\title{
Modeling of Gust Energy Extractions through Aeroelastic Tailoring
}

\author{
by \\ Michael Melville \\ Bachelor of Engineering, Ryerson University, 2016 \\ A thesis \\ presented to Ryerson University

\begin{abstract}
in partial fulfillment of the
Master of Applied Science

in the program of

Aerospace Engineering
\end{abstract} \\ requirements for the degree of
}

Toronto, Ontario, Canada, 2018

(C)Michael Melville 2018 


\section{AUTHOR'S DECLARATION FOR ELECTRONIC SUBMISSION OF A THESIS}

I hereby declare that I am the sole author of this thesis. This is a true copy of the thesis, including any required final revisions, as accepted by my examiners.

I authorize Ryerson University to lend this thesis to other institutions or individuals for the purpose of scholarly research.

I further authorize Ryerson University to reproduce this thesis by photocopying or by other means, in total or in part, at the request of other institutions or individuals for the purpose of scholarly research.

I understand that my thesis may be made electronically available to the public. 


\title{
Modeling of Gust Energy Extractions through Aeroelastic Tailoring
}

\author{
Michael Melville
}

Master of Applied Science, Aerospace Engineering, Ryerson University, Toronto (2018)

\begin{abstract}
A tightly coupled fluid-structure interaction model is presented for studying the performance of flexible wings that encounter atmospheric gusts. The aerodynamic module uses a higher-order potential flow method, that provides numerical robustness and efficiency. The structural dynamics is modelled through an explicit finite difference method of the time-depenedent Euler-Bernoulli equations. Coupled together, these approaches offer numerical accuracy at a fraction of the computational time than is required for higher fidelity approaches. Previous research has suggested energy gains are possible from atmospheric gusts through aeroelastic tailoring. Case studies were performed using the aeroelastic model to investigate the merit of using aeroelastic tailoring as a passive means for performance improvement. Design trends were established that highlight configurations that achieve the best energy extraction from a gust. Reductions in wing drag of between $6.9 \%$ and $10.5 \%$ were observed, while gains of $0.25 \%$ between different aeroelastic configurations were presented. The forward sweeping of the elastic axis was deemed to have the greatest effect on energy extraction capabilities.
\end{abstract}




\section{ACKNOWLEDGEMENTS}

I would first like to thank my thesis supervisors, Dr. Götz Bramesfeld and Dr. Hekmat Alighanbari, for their continued support throughout this research. Their expertise and encouragement helped make the transition to graduate studies both seamless and enjoyable.

I would also like to thank all of my colleagues in the Ryerson Applied Aerodynamics Laboratory of Flight for their always available technical support and words of encouragement throughout this research. 


\section{TABLE OF CONTENTS}

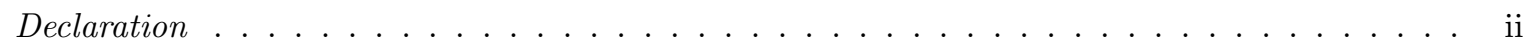

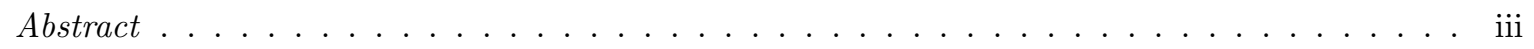

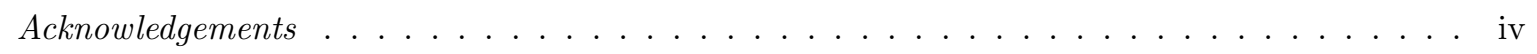

List of Tables . . . . . . . . . . . . . . . . . . . . . . . vii

List of Figures . . . . . . . . . . . . . . . . . . . . . . . . viii

\begin{tabular}{lll}
\hline & Introduction & 1
\end{tabular}

1.1 Fluid-Structure Interaction $\ldots \ldots \ldots \ldots \ldots \ldots \ldots$

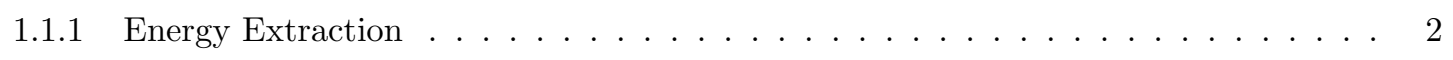

1.2 Numerical Methods . . . . . . . . . . . . . . . . . . . . . . . . . . . . . 5

$1.2 .1 \quad$ Computational Fluid/Structural Dynamics $\ldots \ldots \ldots \ldots \ldots$

$1.2 .2 \quad$ Implicit/Explicit Finite Difference Methods $\ldots \ldots \ldots \ldots$. . . . . . . . . . . . . . 7

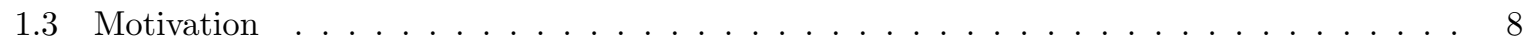

2 Higher-Order Potential Flow Method $\quad 9$

2.1 Alternative Approaches $\ldots \ldots \ldots \ldots \ldots \ldots \ldots$

2.2 Lifting Surface $\ldots \ldots \ldots \ldots \ldots \ldots \ldots$

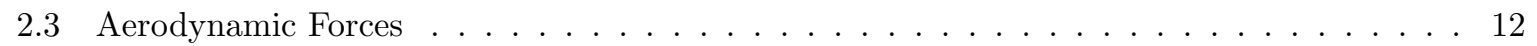

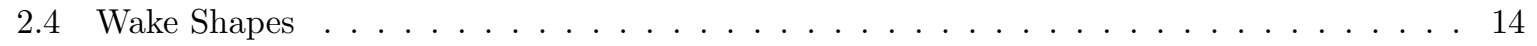

2.5 Unsteady Aerodynamics $\ldots \ldots \ldots \ldots \ldots \ldots$

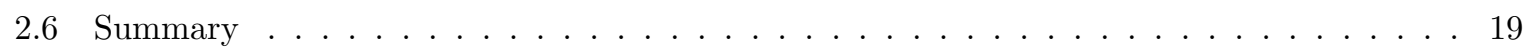

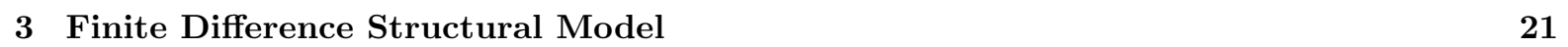

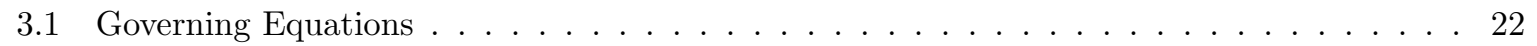

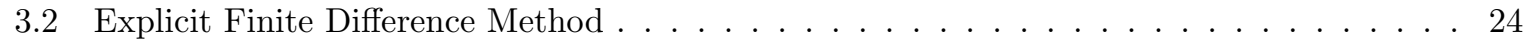

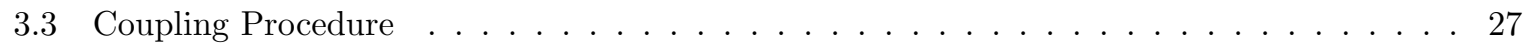


$3.4 \quad$ Numerical Implementation . . . . . . . . . . . . . . . . . . . . . . . . . . . . . . . . . . 29

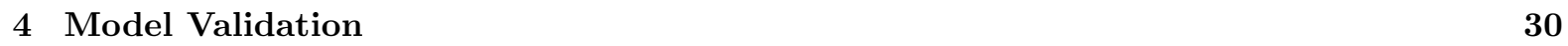

4.1 Static Aeroelastic Response ． . . . . . . . . . . . . . . . . . . . . . . . . . 30

4.2 Modal Analysis . . . . . . . . . . . . . . . . . . . . . . . . . . . 32

4.3 Dynamic Aeroelastic Response $\ldots \ldots \ldots \ldots$

\begin{tabular}{|lll}
5 & Gust Analysis & 39
\end{tabular}

5.1 Gust Implementation $\ldots \ldots \ldots \ldots$

5.2 Application of Aeroelastic Tailoring $\ldots \ldots \ldots \ldots \ldots$

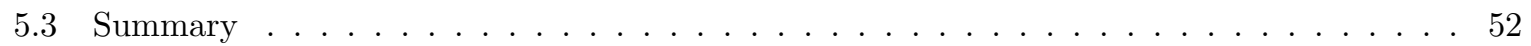

$\begin{array}{lll}6 & \text { Conclusions } & 53\end{array}$

6.1 Aeroelastic Model . . . . . . . . . . . . . . . . . . . . . . . 53

6.2 Implications of Aeroelastic Tailoring $\ldots \ldots \ldots \ldots \ldots \ldots \ldots$

\begin{tabular}{|l|l|}
\hline Appendix A Aerodynamic Module Input File & 55
\end{tabular}

\begin{tabular}{|ll|}
\hline Appendix B Structural Properties Input File & 57
\end{tabular}

\begin{tabular}{ll}
\hline Bibliography & 61
\end{tabular} 


\section{LIST OF TABLES}

4.1 Geometric characteristics for SB-14 aircraft. . . . . . . . . . . . . . . . . . . 31

4.2 Comparison of coupled natural frequencies of Goland wing. . . . . . . . . . . . . . . 34

4.3 Specifications for theoretical wing used for dynamic model validation. . . . . . . . . . . 35 


\section{LIST OF FIGURES}

1.1 NASA Helios Solar UAV during a test flight [2]. . . . . . . . . . . . . . . 2

1.2 Generation of net thrust from plunging airfoil $[6] . \ldots \ldots \ldots \ldots$

1.3 Wake shapes producing a net drag and net thrust $[6] . \ldots \ldots \ldots \ldots$

$1.4 \quad$ Finite difference method grid configurations. . . . . . . . . . . . . . . . . . . . 7

$2.1 \quad$ A distributed vorticity element composed of two vortex filaments and two semi-infinite

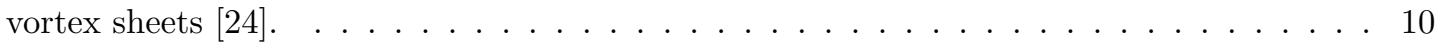

2.2 Six spanwise and two chordwise elements are used to model a wing with geometric twist. 11

2.3 Downwash distribution from Vortex Lattice Method and Distributed Vorticity Elements (Courtesy of Bill Bissonnette). . . . . . . . . . . . . . . . . . . . 12

$2.4 \quad$ Wake generated from a sinusoidal heaving motion $[27] . \ldots \ldots \ldots \ldots$. . . . . . 14

2.5 Comparison between wake shapes of fixed and relaxed wake models. . . . . . . . . . . 15

2.6 Comparison between fixed and relaxed wake computation time per time step. . . . . . . 16

2.7 Comparison of induced drag coefficient in fixed and relaxed wake models. $\ldots \ldots$. . . . . 17

2.8 Sharp-edge gust model. $\ldots \ldots \ldots \ldots \ldots$

2.9 Comparison of quasi-steady and fully unsteady response to sharp-edge gust with 12 spanwise and 6 chordwise elements. . . . . . . . . . . . . . . . . . . . . . . . . . . . 19

2.10 Validation of unsteady aerodynamics module with analytical solution to the Küssner function with varying chordwise elements. . . . . . . . . . . . . . . . . . . . . . . 20

3.1 Coordinate system used for aeroelastic analysis . . . . . . . . . . . . . . . 23

3.2 Discretization used for finite difference structural model. . . . . . . . . . . . . . . . . . . . 24

3.3 Comparison between tightly and loosely coupled approaches [29]. . . . . . . . . . . . . . . 27

3.4 Coupling procedure for dynamic response of elastic wing. . . . . . . . . . . . . . . . . . . 28

4.13 -view of Braunschweig SB-14 high performance sailplane 30 . . . . . . . . . . . 30 
4.2 Static aeroelastic response of the SB14 at $30 \mathrm{~m} / \mathrm{s}$ using an explicit finite difference method. 32

4.3 Static aeroelastic response of the SB14 at $60 \mathrm{~m} / \mathrm{s}$ using an explicit finite difference method. 33

$4.4 \quad$ FFT analysis for free vibration of Goland wing. . . . . . . . . . . . . . . . . 34

4.5 Spanwise loading distribution for test case wing geometry. . . . . . . . . . . . . . 36

4.6 Sensitivity of span efficiency prediction to number of spanwise elements. . . . . . . . . . 37

4.7 Effect of aerodynamic damping on dynamic structural response to sharp-edge gust. . . . . 38

5.1 Implementation of a traveling gust with arbitrary profile $[25] \ldots \ldots \ldots$. . . . . 40

5.2 Incorporation of a gust profile with a VLM $[25] . \ldots \ldots \ldots \ldots$

5.3 Two gust profiles investigated in case studies. . . . . . . . . . . . . . . . . . 41

5.4 Changes to profile and induced drag as a result of traversing through a gust. . . . . . . 42

5.5 Drag reduction with varying chordwise location and orientation of the elastic axis. . . . . 44

5.6 Time history of elastic response and instantaneous drag reduction for $0.3 \mathrm{c}$ swept elastic axis configuration. . . . . . . . . . . . . . . . . . . . . . . . 46

5.7 Dependency of wing drag reduction on lift coefficient. $\ldots \ldots \ldots \ldots$. . . . . . 47

5.8 Time history of force coefficients and elastic deformations at lift coefficients of 0.4 (dashed) and 0.8 (solid). . . . . . . . . . . . . . . . . . . . . . . . . . . . . . . . . . . . . . . . . . . 48

5.9 Time history of force coefficients, elastic deformations and instantaneous drag reduction for two sinusoidal gust profiles, up-down (solid) and down-up (dashed). . . . . . . . . . . 50

5.10 Time response of wing root bending moment for swept elastic axis. . . . . . . . . . . 51 


\title{
LIST OF SYMBOLS
}

\author{
$A, B, C$ Circulation coefficients \\ $C_{D} \quad$ Wing drag coefficient \\ $C_{k} \quad$ Damping matrix \\ $\bar{c} \quad$ Mean aerodynamic chord \\ $c_{m_{0}} \quad$ 2-dimensional zero-lift aerodynamic moment coefficient \\ $D_{\text {ind }}^{\prime} \quad$ Induced drag per unit span \\ EI Bending stiffness \\ GJ Torsional stiffness \\ $h \quad$ Bending displacement \\ $I_{p} \quad$ Polar moment of inertia \\ $K \quad$ Stiffness matrix \\ $L^{\prime} \quad$ Lift force per unit span \\ $L_{G} \quad$ Gust length \\ $l_{s m} \quad$ Distance between shear and mass centers \\ $M \quad$ Mass matrix \\ $M^{\prime} \quad$ Aerodynamic moment per unit span \\ $w_{0} \quad$ Gust magnitude
}




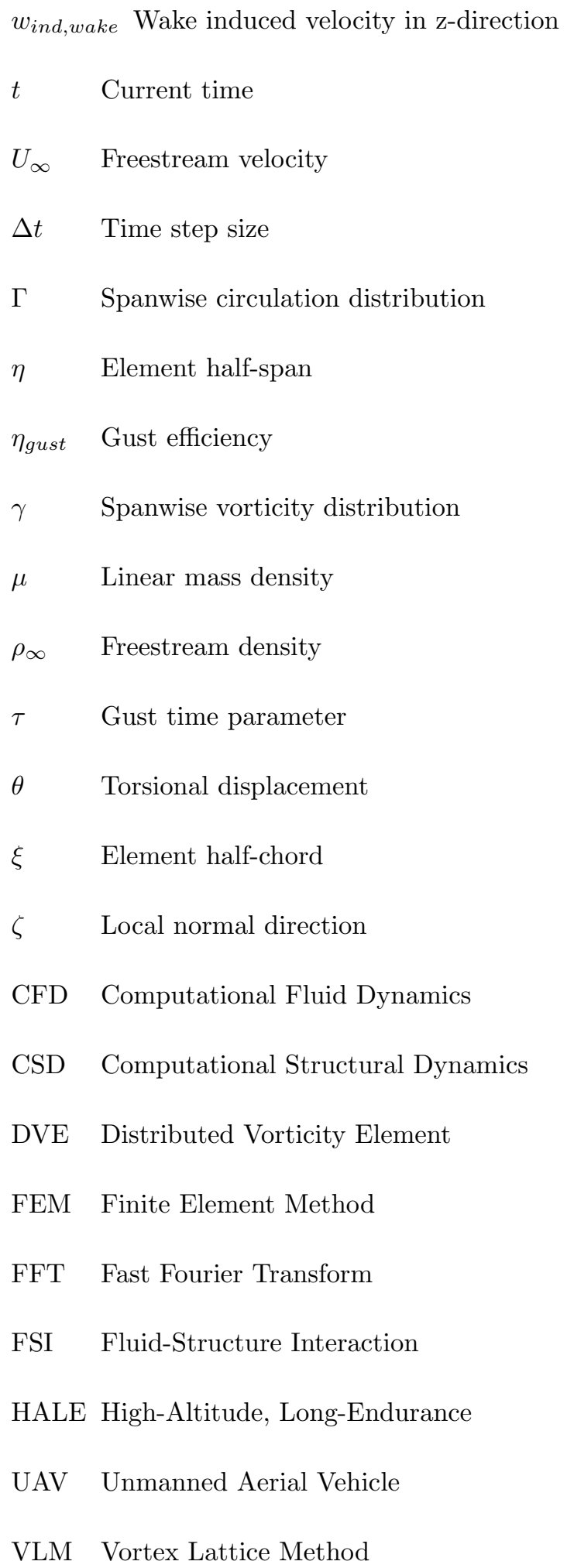




\section{Chapter 1}

\section{Introduction}

\section{$1.1 \quad$ Fluid-Structure Interaction}

The study of fluid-structure interaction (FSI) looks to investigate the elastic response of a structure due to an applied, often time varying, load. FSI problems can manifest themselves in many fields of engineering including civil, mechanical and aerospace. The coupling between these external loads and the structural response is a product of both the structural properties and the nature of the applied load. Under certain conditions, the aeroelastic frequencies in both torsion and bending can approach one another; this is a source of unstable behaviour, as the coalescing of these frequencies results in large amplitude oscillations. The effects of these interactions can create catastrophic results if not properly accounted for. A very common example of an FSI problem is seen in civil engineering with the Tacoma Narrows bridge. The excitation of the torsional mode of vibration caused large amplitude oscillations, eventually resulting in the failure of the bridge. Research by Arioli and Gazzola [1] indicates that the failure was a combination of both the aerodynamics from the vortex streets being shed from the bridge and the structural characteristics; an equilibrium state was reached, in which the input energy from the aerodynamics was balanced by the internal dissipation of the structure, allowing the large amplitudes to be maintained.

A more applicable example to the aerospace industry is the failure of the NASA Helios Solar Unmanned Aerial Vehicle (UAV), pictured in Fig. 1.1. The Helios was a high aspect ratio, very flexible aircraft, fitted with solar cells across the entire upper wing surface. During a test flight, the aircraft encountered turbulence which caused the aircraft to transition to a high dihedral configuration, due to the very flexible structure. The aircraft, as a result, became unstable and experienced rapidly growing 
pitch oscillations. This caused aerodynamic loads to increase beyond design conditions, and the wing structure subsequently failed [2].

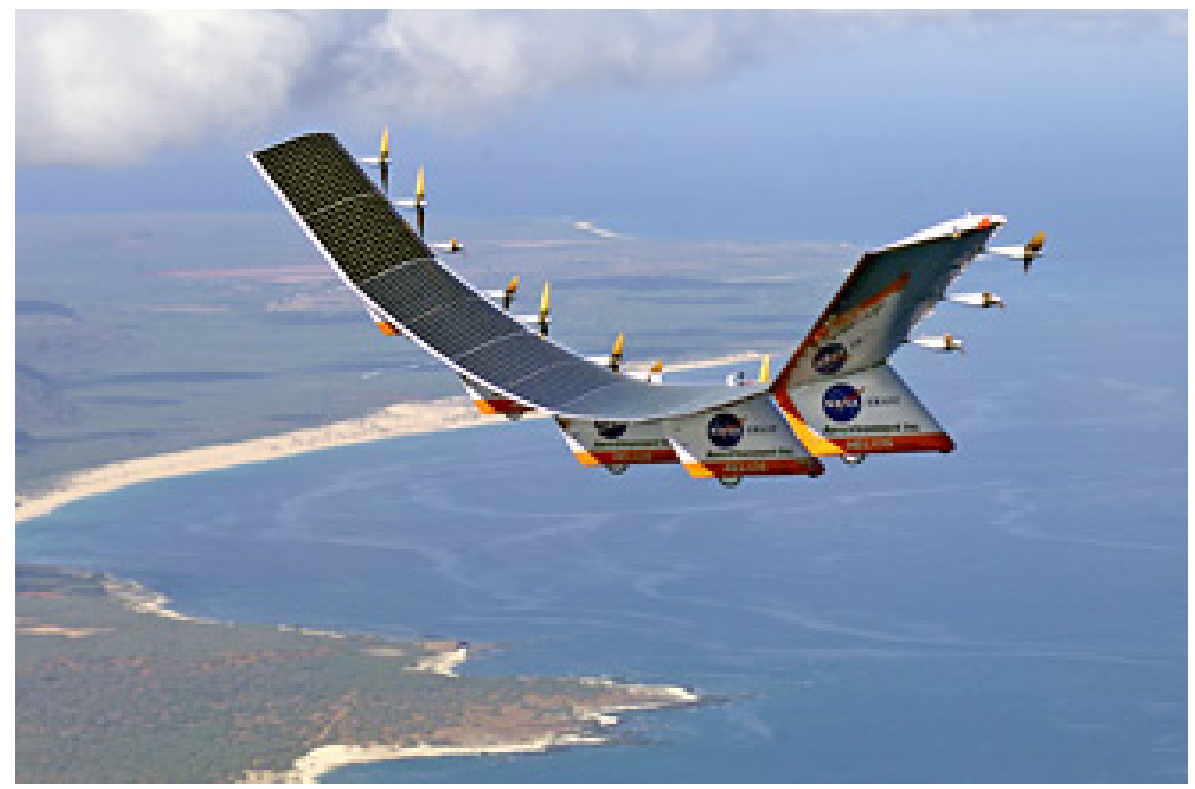

Figure 1.1: NASA Helios Solar UAV during a test flight [2].

The dynamic structural response to an applied load is often a combination of both bending and twisting. This coupling is a result of the relative location of the elastic axis with respect to the mass center of the structure [3]. In static aeroelasticity, the placement of the elastic axis relative to the aerodynamic center also plays a significant role in the aeroelastic stability of the system. Placing the elastic axis too far aft of the aerodynamic center can make the wing prone to divergence; divergence occurs when the twist response of the wing becomes uncontrolled and increases rapidly, eventually leading to structural failure.

\subsubsection{Energy Extraction}

Elastic deformations of aircraft wings are often thought of as a negative consequence to flight loads; deflection of the wing creates reductions in effective span, resulting in an increase in induced drag on the aircraft [4. However, it is possible to extract performance gains from these deflections, specifically when encountering a gust. This is due to a phenomenon known as the Katzmayr or Knoller-Betz effect. Katzmayr [5] investigated the effects of periodic changes in angle of attack on an airfoil through experimental wind-tunnel testing. It was found that a net thrust could be obtained as a result of these periodic changes. This net thrust is a result of the tilting of the lift vector as the effective angle of 
attack is changed; this phenomenon is demonstrated in Fig. 1.2. As the gust approaches from below, an increase in effective angle of attack is seen, tilting the resultant force vector forward. Decomposing this force, there is a lift component perpendicular to the freestream, $U_{\infty}$, and an additional forward component, opposite to the drag direction, which can be attributed to the net "thrust".

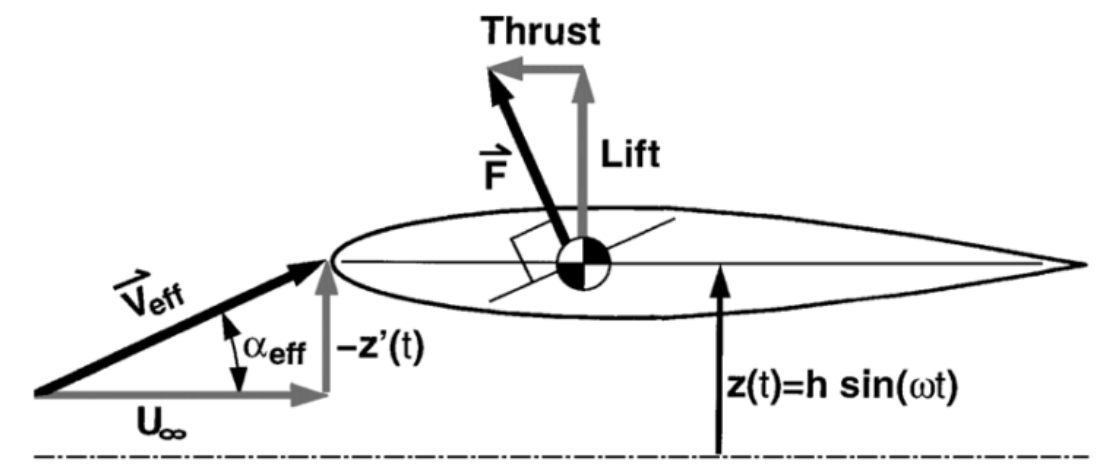

Figure 1.2: Generation of net thrust from plunging airfoil [].

An extension of this research was later performed by Jones et al. [6], in which both experimental and computational models were developed in order to demonstrate possible energy gains from a plunging airfoil. Using flow visualization techniques, the experimental models investigated wake profiles that are indicative of a net thrust being produced. The numerical model used an unsteady panel method. Wake velocity profiles from this model were compared to those of the experimental results. The research by Jones et al. provided insight into reduced frequencies that provide a net "thrust" and the corresponding wake structures. An example of drag and thrust producing wake structures are shown in Fig. 1.3a and Fig. $1.3 \mathrm{~b}$ respectively. The difference between drag and thrust producing wakes is dependent on the plunge velocity of the airfoil. Lower plunge velocities create a drag producing wake as the distance between vortices of like rotation directions in the vortex street is small. As the plunge velocity is increased, the distance between these vortices becomes larger, producing a wake structure that is indicative of a net thrust.

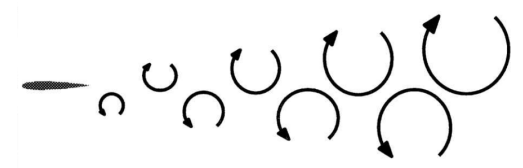

(a) Drag producing wake structure

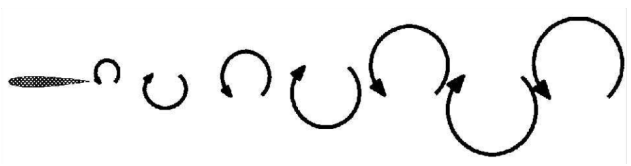

(b) "Thrust" producing wake structure

Figure 1.3: Wake shapes producing a net drag and net thrust [6]. 
While the aforementioned research was done only for 2-dimensional airfoils, the same phenomenon can be extended to a finite, flexible wing. The motion of a wing section undergoing elastic deformation is analogous to that of a plunging airfoil; a local change in effective angle of attack is experienced, similar to that shown in Fig. 1.2, due to the induced velocity of the upwards or downwards motion of the wing. Consequently, it is possible to extract this "thrust" as a result of elastic deformations. The motion induced as a result of the wing deflection, coupled with the addition of a gust velocity can translate to significant drag reductions (i.e. "thrust" gains) for an aircraft. Mai [7] performed a detailed investigation into energy retrieval from gusts of a flexible sailplane model, where the entire aircraft was modelled and allowed to displace as a result of the gust. The study concluded that the bending of the wing played a much more significant role in energy retrieval from the gust than that of the torsional response. This research was among the first to investigate the effects of FSI on a flexible aircraft, demonstrating the need for more detailed studies.

While it is clear that energy extraction from a gust is possible through elastic deformations, one must also consider at what scale these gains are significant. Phillips [8] studied how the propulsive effects of flight through a turbulent atmosphere affect aircraft of 4 different sizes. It was found that for light aircraft and sailplanes, the thrust gains were an order of magnitude larger than those for fighter and transport aircraft. This indicates further studies should be limited to smaller scale, low speed aircraft in order to truly investigate the effects of gust energy retrieval.

\section{Aeroelastic Tailoring}

Several active forms of gust energy extraction exist that involve the use of added control systems and on-board equipment. For instance, Bruni et al. 9] investigated how adding piezoelectric elements to a wing can affect the energy extraction; modification of the internal resistance of these elements affects the scale to which energy may be harvested. Langelaan [10] investigated how feedback control laws can be used to harvest energy from gusts for mini and micro UAVs. Such active methods can be difficult to implement and properly tune; the addition of extra components can also translate into unwanted added mass.

A passive method to improve gust energy extraction is through aeroelastic tailoring. Aeroelastic tailoring involves carefully tuning the stiffness and mass distributions across a wing in order to achieve a desired structural response [11. Through this tuning, the mass center and elastic axis distributions can be modified, thus altering the coupling between the aerodynamics and structural dynamics. Aeroelastic tailoring is common in composite wing structures, where simply altering fiber orientations can achieve a desired bending-torsion coupling. The benefits of aeroelastic tailoring have been investigated by many 
researchers including works by Weisshaar [12, Lupp [13, and Francois et al. 14]. Weisshaar [12] showed possibilities of induced drag reductions through aeroelastic tailoring. This result is further exemplified through the work of Lupp et al. [13, in which a sailplane structure was optimized in order to maximize the average cross-country speed. While only a small percentage increase in average cross-country speed was seen, even small improvements can translate into large gains, specifically in sailplane design. Francois et al. 14] showed various ways in which aeroelastic tailoring may be implemented, citing structural weight savings when using various rib/spar orientations. It is clear that aeroelastic tailoring has been demonstrated to show possible performance improvements with little to no mass penalties.

\subsection{Numerical Methods}

There are several existing methods for addressing FSI problems ranging from high fidelity methods such as coupled Computational Fluid Dynamics (CFD) and Computational Structural Dynamics (CSD), to lower fidelity ones such as potential flow and finite difference methods. Higher fidelity methods can provide improved solution resolution, however, at the cost of a significant increase in computational expense. Lower fidelity methods, while not as accurate as higher fidelity ones, can be used much more rapidly, while still offering adequate solution accuracy. The use of each of the above approaches is dependent on the needs of the designer; lower fidelity methods are very useful in early design stages as they help to eliminate designs that do not meet the set criteria, while higher fidelity methods may be used at a later stage of the design process in order to perform more detailed investigations and fine tune the design. Several existing approaches in both the high and low fidelity regime are discussed further below.

\subsubsection{Computational Fluid/Structural Dynamics}

Computational fluid dynamics is a very commonly used approach for modelling aerodynamics in industry and academic research applications. The high-fidelity nature of CFD often provides the user with results that closely match experimental data. When investigating fluid-structure interactions from a high-fidelity perspective, CFD models are typically coupled with CSD modules; this allows for accurate resolution of the aerodynamic and structural dynamic behaviour. The degree to which these models are coupled is broken down into three different classes: fully-coupled, tightly-coupled and loosely-coupled [15. Fully-coupled methods combine the aerodynamic and structural dynamic equations to form a single set of equations that is solved. Tightly-coupled models keep the aerodynamic and structural dynamic governing equations separate; information between each is transferred between time steps in the form of 
aerodynamic loads and elastic deformations. Loosely-coupled approaches limit the interactions between CFD and CSD solvers; information is usually only updated after certain convergence criteria is met. The approaches presented here are in order of most computationally expensive to least; the computational cost is a function of both the overhead required for solving the equations of motion as well as the need for constant updating of the grid position. Fully-coupled and tightly-coupled require the aerodynamic grid to be updated each time step as the structure is deformed; loosely-coupled models do not require as frequent updates and thus are typically more computationally efficient, at the expense of a loss in accuracy.

Several approaches using coupled CFD-CSD models have been used to investigate the aeroelastic behaviour of both entire aircraft or just their wing systems. Smith et al. [15] used a coupled Euler/NavierStokes solver coupled with a non-linear structural model in order to investigate the aeroelastic behaviour of a theoretical High-Altitude, Long-Endurace (HALE) wing. Their research demonstrated the accuracy of these higher-fidelity methods by comparing the results to those from a panel method; an overprediction from the panel method was noted. Raveh [16] used a CFD-based method for modeling the gust response of a flexible aircraft. The investigation was performed for steady-level flight of a transonic aircraft using two different implementations of the gust model. The direct approach presented in the paper improved the accuracy of the results, although, at a significant computational cost. The presented hybrid approach provided reduced accuracy, but a significant reduction in computation time. Reimer et al. 17. provided a comparison of a CFD-based analysis to a lower-fidelity Doublet-Lattice Method (DLM) when investigating gust loads on a flexible aircraft. The study showed that the DLM solver provided an over-prediction in load-factors in comparison to CFD across both transonic and subsonic regimes; this is likely a result of an over-prediction in lift due to the use of a panel code. The addition of thickness creates a steeper lift curve slope for the lifting surface; this increase is typically negated by viscous effects, however, these are not considered in a potential flow approach.

Several studies have been conducted in comparing coupled CFD-CSD approaches to lower fidelity models, such as those illustrated above. The results from these studies are consistent in showing the improved accuracy that the high-fidelity CFD-based approaches offer. One must be cautious, however, in the selection of such methods. The improved accuracy often comes at the expense of a significant increase not only in computational time, but in pre and post-processing work. The need for creating refined meshes for both the aerodynamic and structure domain is something that is often overlooked for CFD-based analysis. Post-processing of results can also be difficult, since only the "big picture" is provided (i.e. total lift and total drag). Additional work is required to decompose the results to determine the largest contributors, which is often more important than the final numbers themselves. 


\subsubsection{Implicit/Explicit Finite Difference Methods}

Finite difference relationships are based off of approximations to higher order derivatives; using a series expansion, a simple fractional relationship that uses solutions at other "node" points is used in order to approximate a derivative. These relationships are very useful for modeling the differential form of governing equations, such as the dynamic Euler-Bernoulli beam equations [18. There are two basic forms to finite difference methods known as explicit and implicit. The basic principle of these finite difference methods is shown in Fig. 1.4. Explicit methods utilize the solution at multiple grid points at the past time step (say time $t$ ) in order to determine the solution at a single node at the current time step (time $t+\Delta t$ ), shown in Fig. 1.4a. Ironside et al. [19] used such an approach model the aeroelastic response of a sailplane wing configuration. The advantage of the explicit approach is its simplicity to implement and relatively low computational cost; it can, however, be subject to stability issues at larger time step sizes.

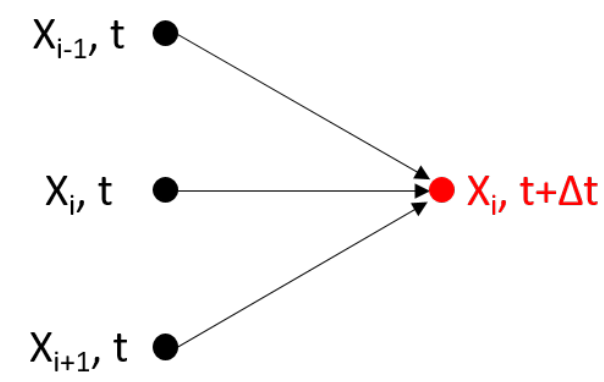

(a) Explicit finite difference method

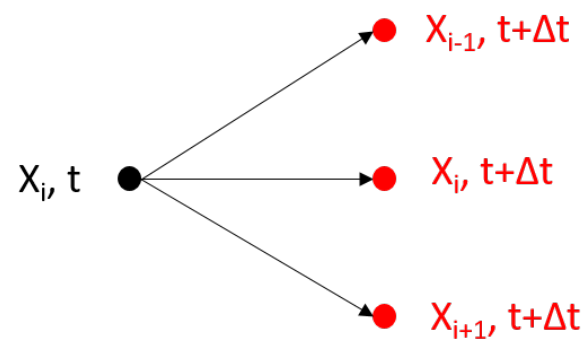

(b) Implicit finite difference method

Figure 1.4: Finite difference method grid configurations.

Implicit methods are implemented in a slightly difference fashion in comparison to explicit methods; these methods utilize the solution at one node point in the past to solve for the solution at multiple nodes at the current time, as seen in Fig. $1.4 \mathrm{~b}$. This leads to the need for a matrix solution where the solution at all node points for the current time are solved concurrently. Subbaraj and Dokainish [20] provided an investigation into multiple implicit approaches that may be used for structural dynamics. Among these include the Newmark family of methods [21, the Wilson- $\theta$ method [22] and the Houbolt method [23. The investigation provided in Ref. 20] highlights the robustness of several of these implicit methods. One of the major advantages of these approaches is their unconditional stability, provided certain criteria are met. This implies that given that a certain combination of grid spacing and time step size, a stable solution will always be achieved. The Houbolt method is a very popular example of the use of finite difference methods in aeroelastic analysis. The implicit method developed here was 
applied to the analysis of a flexible aircraft passing through a gust, demonstrating the capability of using large time step sizes, while still producing an accurate result. A disadvantage with implicit approaches, however, is their increased computational cost. The need to store information at several grid points concurrently can create large increases in memory requirements that are not present in explicit methods.

\subsection{Motivation}

This thesis focuses on the development and application of an efficient aeroelastic solver in order to investigate the gust energy extraction capabilities of flexible wing structures. Previous research projects focusing on aerodynamic analysis have indicated drag reductions are possible when encountering a gust. Including the effects of elastic deformations, aeroelastic tailoring can be used as a passive way to maximize these energy extractions.

The solver couples an existing higher-order potential flow method with an explicit finite difference method in order to solve the structural dynamics equations of motion. The higher-order potential flow method uses elements with distributed vorticity rather than vortex filaments that are common to other approaches; the use of distributed vorticity allows for the avoidance of many of the singularity issues that exist with these other, more conventional methods. This approach also offers a computationally inexpensive means for resolving induced drag changes, which are the driving force in this analysis. The explicit finite difference method provides an efficient means for computing the dynamics of the structure, while still maintaining a high order of accuracy.

The nature of this coupled solver makes it an attractive approach for the use in early design stages. Complex interactions can still be modelled through the higher-order method, while providing a simple means to decompose the results in order to get a true picture of the major contributors to the gust energy extraction.

In order to determine how elastic deformations may be used for energy extraction, case studies were performed to investigate the effects of varying the elastic axis and center of mass locations on the wing. The current method allows for these properties to be easily interchanged, without the need to regenerate the computational domain. The results from these case studies help to provide insight into this passive form of energy extraction and provide designers with knowledge on which configurations perform best. 


\section{Chapter 2}

\section{Higher-Order Potential Flow Method}

The aerodynamics of the presented aeroelastic solver is modelled using the higher-order potential flow method developed by Bramesfeld 24]. This method utilizes elements of distributed vorticity (DVEs) to model the lifting surface; the use of such elements aid in improving the numerical robustness of the method. Compared to conventional panel or vortex lattice methods, the use of DVEs also allows one to use fewer spanwise elements to accurately model the circulation distribution and estimate induced drag.

\subsection{Alternative Approaches}

There are several approaches that may be used when seeking to model aerodynamics through potential flow. Conventional methods include the Vortex Lattice Method (VLM), panel method, and lifting line method. Vortex lattice methods use a series of vortex filaments of constant strength to model the lifting surface. The method allows for both spanwise and chordwise elements, providing accurate force resolution. A disadvantage with this approach is the singularities associated with the vortex elements, specifically in the wake (i.e. vortex filaments). These elements induce a velocity that becomes infinity as one approaches the core. The constant strength of these filaments requires that several elements across the span be used in order to resolve induced drag accurately; the use of fewer elements creates a step-wise circulation distribution through the wake, resulting in poor force resolution. VLM approaches can also be adapted to model unsteady behaviour, such as that seen in Ref. 25.

Panel methods are similar to VLM approaches in that they use a distribution of constant strength 
vortex filaments to model the lifting surface. Unlike VLMs, panel methods are capable of modelling thickness. This, however, typically causes an over-prediction in lift since the addition of thickness creates a steeper lift-curve slope. With the use of vortex elements, panel methods are also prone to numerical instabilities, which can limit their range of application.

Often, lifting-line methods model the bound circulation distribution across the wing by placing a vortex filament along the quarter-chord line. Multiple bound vortex systems maybe be used across the span to improve the accuracy of the force distribution, however, no chordwise information is captured. Traditionally, lifting-line methods are limited to relatively simple geometries, such as straight wings with no sweep. The wakes generated through this method are often continuous vortex sheets which induced finite velocities everywhere except in the sheet itself. Improved numerical robustness is seen in this approach, however, one is limited to a fixed wake.

\section{$2.2 \quad$ Lifting Surface}

As previously mentioned, the lifting surface in the herein presented results, was modelled using distributed vorticity elements. These elements, as shown in Fig. 2.1, consist of vortices along the leading and trailing edge that have parabolic circulation distributions. The local reference frame is in the spanwise and chordwise direction, represented by $\eta$ and $\xi$, respectively.

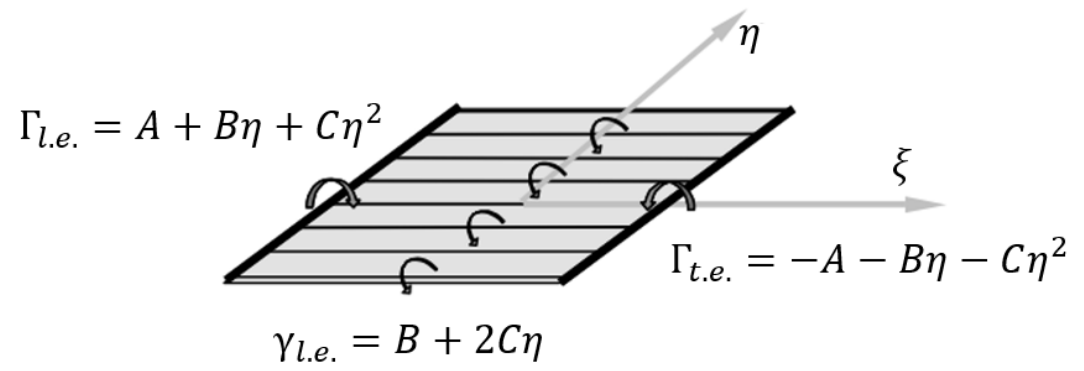

Figure 2.1: A distributed vorticity element composed of two vortex filaments and two semi-infinite vortex sheets [24].

The spanwise circulation distribution, $\Gamma$, of the leading edge vortex filament follows the form:

$$
\Gamma(\eta)=A+B \eta+C \eta^{2}
$$

where $\eta$ is the element half-span coordinate, and $A, B$, and $C$ are the circulation coefficients. The vortex filaments along the leading and trailing edges of the element have equal, but opposite strengths, 
as shown in Fig. 2.1. A streamwise vortex sheet connects the leading and trailing edge vortex filaments. That vortex sheet has linear spanwise vorticity distribution, $\gamma$ :

$$
\gamma(\eta)=\frac{d \Gamma}{d \eta}=B+2 C \eta
$$

where $B$ and $C$ are the same circulation coefficients from Eq. 2.1.

The distributed vorticity elements are placed along the zero-lift plane of the wing. The elements remain connected at their leading edge and are then rotated about here to model geometric twist across the span, as shown in Fig. 2.2 The formulation of Eq. 2.1) allows for a continuous circulation distribution across the span that follows a second-order spline. Consequently, significantly fewer spanwise elements are required in comparison to similar VLM approaches, to achieve a similar force resolution 24 .

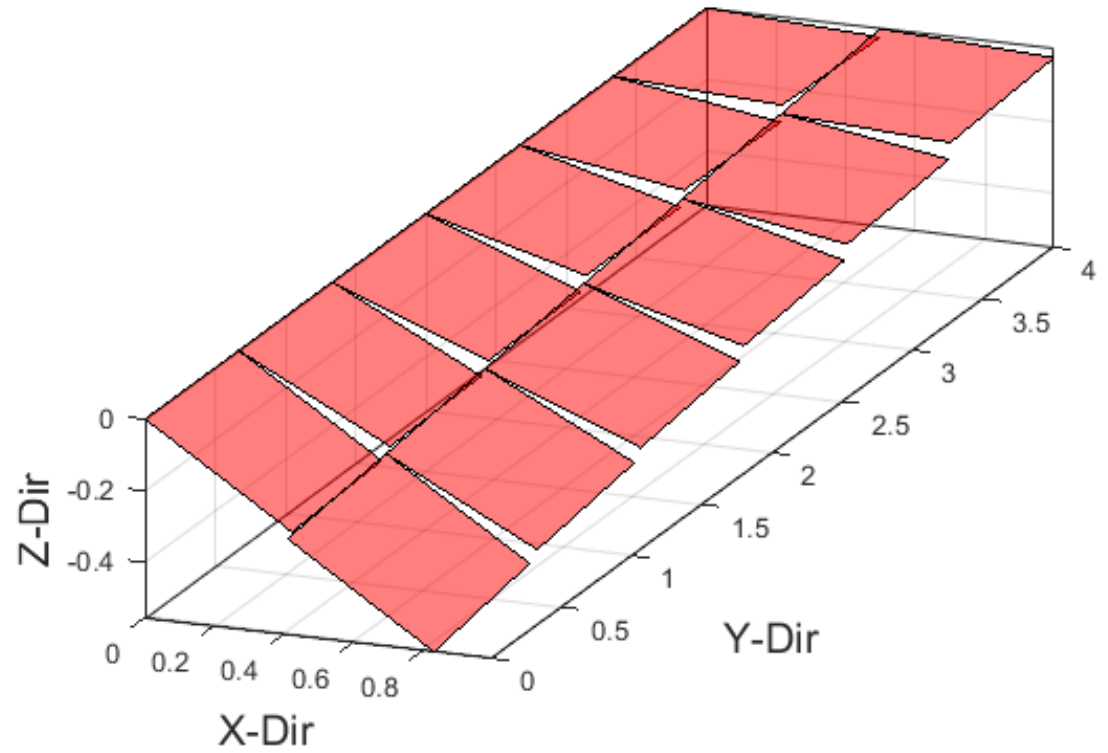

Figure 2.2: Six spanwise and two chordwise elements are used to model a wing with geometric twist.

In order to obtain a better resolution of aerodynamic forces, multiple elements across the span and chord may be used. The use of chordwise elements becomes especially important when investigating unsteady behaviours, which is further explored in Section 2.5 .

The wake is developed through a time-stepping procedure using the same distributed vorticity elements; the circulation on the lifting surface at each time step is shed from the trailing edge into the wake. 
For steady analysis, the total circulation shed at each spanwise location is applied to all wake elements; thus, no vortex filaments are present in the wake and it can be modelled as a continuous vortex sheet. This eliminates several singularity problems that are common to other approaches, producing finite wake induced velocities everywhere. Figure 2.3 shows a comparison of the downwash distribution created from a conventional VLM and the described distributed vorticity elements. As one approaches the vortex filaments, the induced velocity from the VLM becomes singular; the use of distributed vorticity elements creates a continuous downwash distribution across the wing span. This continuous formulation allows for induced drag to be computed efficiently with very few spanwise elements in comparison to traditional potential flow methods.

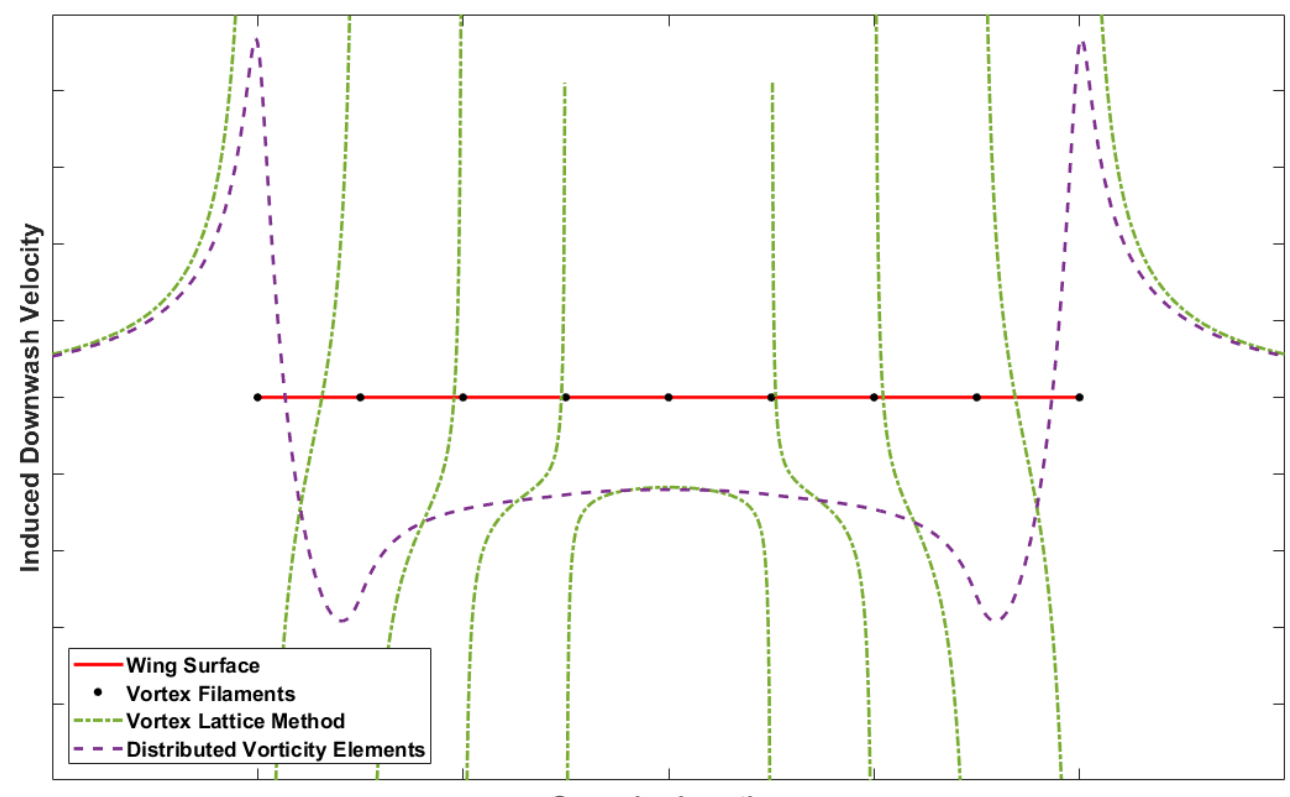

Spanwise Location

Figure 2.3: Downwash distribution from Vortex Lattice Method and Distributed Vorticity Elements (Courtesy of Bill Bissonnette).

\subsection{Aerodynamic Forces}

Aerodynamic forces are computed by solving a system of linear equations for the circulation coefficients, $A, B$, and $C$, at each surface element and then applying the Kutta-Joukowsky theorem. The system of 
equations follows the form:

$$
\mathbf{D} \cdot \underline{\Gamma}=\underline{R}
$$

where $\mathbf{D}$ is an influence matrix which is dependent on the geometry of the lifting surface, $\underline{\Gamma}$ is the desired circulation coefficients and $\underline{R}$ is the resultant which enforces the boundary conditions.

Four different boundary conditions across the lifting surface are applied. The first two boundary conditions specify that circulation and vorticity must be continuous across the boundary of each DVE. That is to say $\Gamma_{1}=\Gamma_{2}$ and $\frac{d \Gamma_{1}}{d \eta}=\frac{d \Gamma_{2}}{d \eta}$. Additional boundary conditions are applied at the wing root and tip to facilitate accurate modelling of the circulation distribution. A condition of zero circulation and undefined vorticity is applied at the wingtip; this ensures that the lift force is zero at the wingtip. At the wing root, a symmetry boundary condition of constant vorticity (i.e. $\gamma=\frac{d \Gamma}{d \eta}=0$ ) is applied; this enforces zero slope on the circulation distribution, requiring only half of the wing span to be modelled. Furthermore, flow tangency at each of the element control points; the control point is located at the element mid-point, where $\eta=0$ and $\xi=0$.

Lift forces and aerodynamic moments are computed per unit length in order to facilitate integration with the structural dynamics module described in Chapter 3. Lift is computed via:

$$
L^{\prime}=\rho_{\infty} U_{\infty} \times \Gamma
$$

while the aerodynamic moment is a summation of the airfoil pitching moment and lift induced pitching moment:

$$
M^{\prime}=M^{*}+q_{\infty} \bar{c}^{2} c_{m_{0}}
$$

$M^{*}$, is the moment per unit span about the reference axis; for aeroelastic analysis, this is the wing elastic axis. The contributions of each chordwise lifting line are accounted for in this term, since their location relative to the reference axis will vary. The second term implements a strip-theory method to determine the pitching moment created as a result of the section airfoil characteristics. The mean aerodynamic chord, $\bar{c}$, of the section is used as the reference moment arm and the two-dimensional zero-lift pitching moment coefficient, $c_{m_{0}}$, is used as the non-dimensional term.

Induced drag is computed from the circulation distribution present in the wake by applying the Kutta-Joukowsky theory at the lifting surface trailing edge:

$$
D_{\text {ind }}^{\prime}=\rho_{\infty} w_{\text {ind,wake }} \times \Gamma_{\text {t.e. }}
$$


where $\Gamma_{t . e .}$ is the circulation at the trailing edge filament and $w_{\text {ind,wake }}$ is the wake induced velocities here. This a robust approach for computing induced drag which agrees well with Trefftz plane analysis and Euler results [26.

\subsection{Wake Shapes}

The time stepping method for generating the wake allows for two different types of wake shapes. The first is a fixed wake. In the case of the fixed wake model, the wake elements are place along the path of the wing's trailing edge. Thus, a highly non-planar wake can be modelled through this approach, as shown in Fig. 2.4. This figure shows a wing experiencing a sinusoidal heaving motion, with the lifting surface in grey and wake elements in blue. The wake elements provide a trace of the wing's trailing edge position as it follows this heaving motion, producing a wake pattern that follows the same sinusoidal profile.

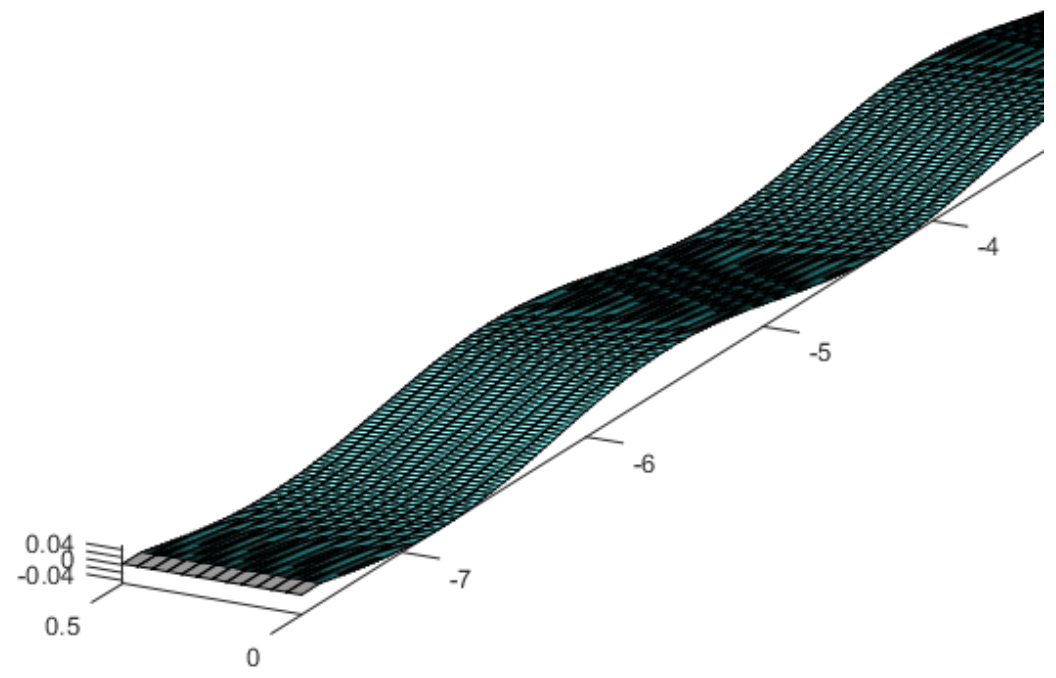

Figure 2.4: Wake generated from a sinusoidal heaving motion [27].

The second wake model that can be used in this method is a relaxed wake. In the case of the relaxed wake model, wake elements are allowed to deform based on the locally induced velocities as shown in Fig. 2.5b As the wake elements are stretched and compressed, their circulation must be adjusted in order to satisfy the Helmholtz theorem, which states that the total circulation in an irrotational flow field must remain unchanged. 
A comparison between the shape of the fixed and relaxed wake models is shown in Fig. 2.5. A tapered wing was moved at a constant angle of attack for 20 time steps; the resulting wake structure is shown by the blue elements, while the lifting surface is shown by the red elements. One of the defining differences between the two models is the ability of the relaxed wake model to capture the effects of the wing tip vortex (see Fig. 2.5b). The wake elements at and near the wing tip experience larger induced velocities from one another and as a result begin to stretch and roll up.

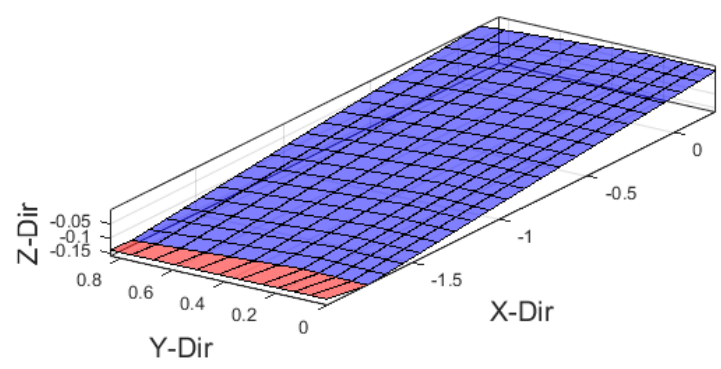

(a) Wake shape generated using fixed wake model

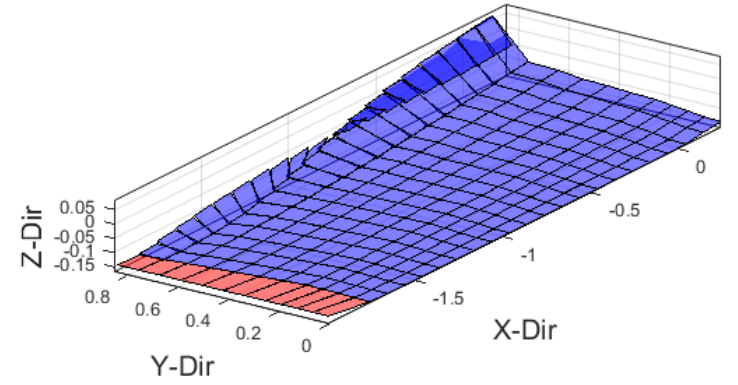

(b) Wake shape generated using relaxed wake model

Figure 2.5: Comparison between wake shapes of fixed and relaxed wake models.

Modelling the roll-up behaviour, however, comes at the cost of a significant increase in computational time. With the fixed wake model, induced velocities from the wake only need to be computed at the lifting surface; thus, computational time is mainly a function of the number of surface elements, assuming the number of time steps is held fixed. In the relaxed wake model, induced velocities need to be computed both on surface and wake elements; computational time increases exponentially as the number of wake elements increases. Figure 2.6 shows a comparison in the computational time required at each time step using the fixed and relaxed wake models. A simple wing geometry was used for this case at a constant angle of attack of $5^{\circ}$, running steady aerodynamics. At more than ten time steps, the difference in computation time that is required between the two wake models begins to become noticeable, and increases exponentially with the total number of time steps.

A comparison of prediction results from the two methods was made by performing an angle of attack sweep from 0 to 10 degrees on the same wing geometry. The span efficiency calculated between the two methods is compared since this is greatly influenced by the wake shape. Figure 2.7 shows that at low angles of attack, there is minimal difference between the two, but as the angle of attack is increased, 


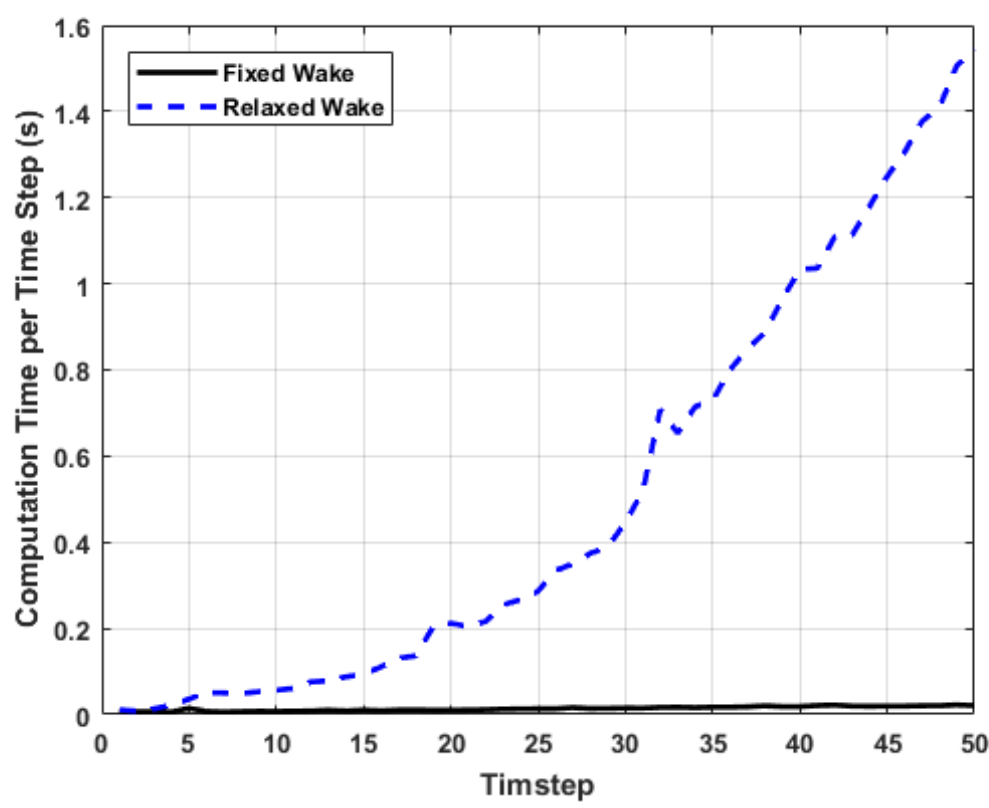

Figure 2.6: Comparison between fixed and relaxed wake computation time per time step.

the offset becomes more apparent. At the highest angle of attack, a 5\% difference is noted between the two methods; this is due to the increased wake roll-up at higher lift coefficients. This $5 \%$ improvement with the relaxed wake model, however, comes at a significant increase in computational time, as shown in Fig. 2.6. Thus, all computations performed moving forward were done using the fixed wake model, as the marginal improvement in performance prediction does not outweigh the increase in computational cost.

\subsection{Unsteady Aerodynamics}

Two different approaches were explored for modelling unsteady behaviour, namely a quasi-steady and fully unsteady model. The quasi-steady model was originally implemented by Bramesfeld [24], and as later adapted to a fully unsteady model by Cole 28. Unsteady aerodynamics from a potential flow perspective, is conventionally derived from the unsteady Kutta-Joukowsky theorem. The total force per unit span, $\bar{F}_{\zeta}^{\prime}$, in the local normal direction, $\zeta$, can be described as:

$$
\bar{F}_{\zeta}^{\prime}=-\rho \int_{0}^{c}\left[\left(u(t) \cos \left(\alpha^{*}\right)-w(t) \sin \left(\alpha^{*}\right)\right) \cdot \gamma_{\xi}+\frac{\partial \Gamma(\xi, \eta, t)}{\partial t}\right] d \xi
$$

The first term represents the lift as a result of the chordwise vorticity distribution, and includes the 


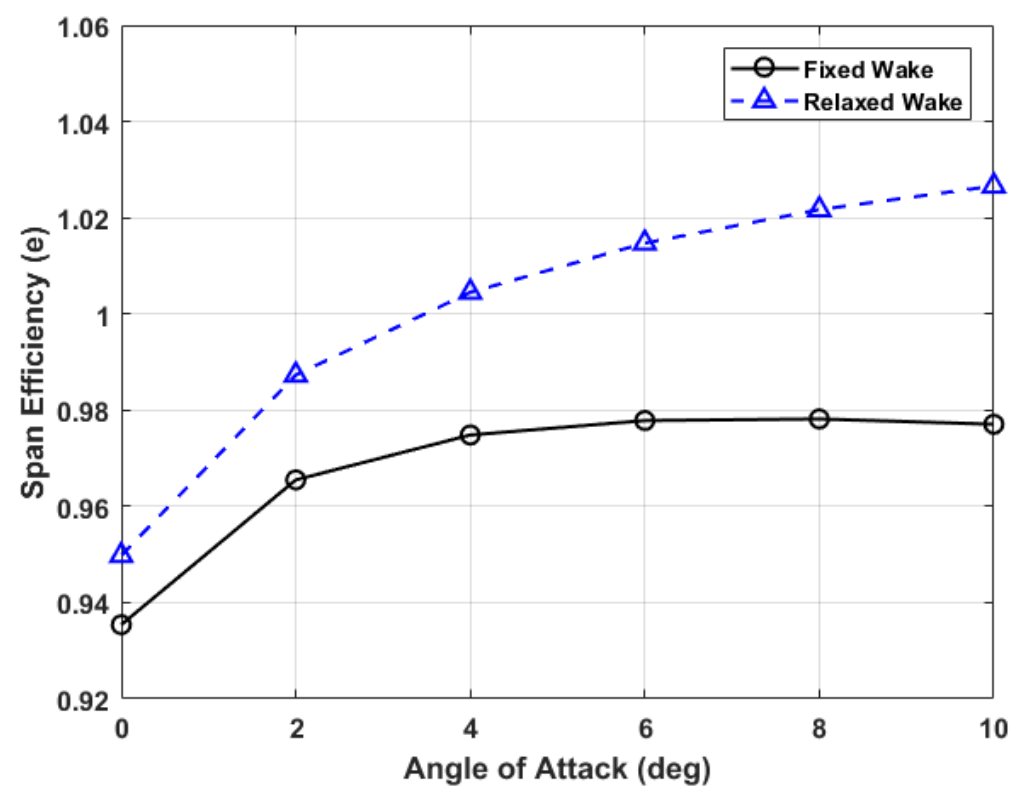

Figure 2.7: Comparison of induced drag coefficient in fixed and relaxed wake models.

effects of circulation being shed into the wake. The components freestream and induced velocities, $u(t)$ and $w(t)$, are accounted for based on their angle, $\alpha^{*}$, from the lifting surface. The chordwise vorticity distribution is accounted for via $\gamma_{\xi}$. The second term of the integrand arises from "non-circulatory" effects and is often referred to as "apparent-mass".

The quasi-steady aerodynamic model incorporates only the first term of Eq. 2.7), capturing the changing circulation shed from the lifting surface into the wake. Unlike for the steady approach, the circulation shed from each spanwise station at each time step is not applied throughout the entire wake; consequently, with quasi-steady aerodynamics, vortex filaments will be present in the wake. While with this approach the wake will contain a time history of the circulation changes on the lifting surface, it presents numerical issues as singularities can be encountered when filaments intersect one another.

The fully unsteady approach implemented by Cole, and captured by Eq. 2.7), accounts for both the shed circulation as well as the apparent-mass term. This apparent-mass term provides a damping effect to lift production on the surface as it accounts for the time rate of change in circulation at each surface element. This allows for a more accurate prediction of highly transient phenomenon where rapid changes in lift are expected. For instance, a fully unsteady approach is required to accurately resolve the response of a wing encountering a gust. The rapid changes in effective angle of attack when encountering a gust result in a circulation distribution that is highly time dependent. 
A case study was performed to analyze the response of a two-dimensional airfoil encountering a sharpedge gust, as depicted in Fig. 2.8. A sharp-edge gust is defined as a sudden increase in the velocity component perpendicular to the direction of travel. This creates a rapid increase in lift in a short period of time, and thus requires an unsteady approach to properly model. The Küssner function exists as an analytical solution for the two-dimensional lift response of an airfoil encountering a sharp-edge gust and thus, provides a point for validation. Thus, the potential flow method was adapted to model a two-dimensional case. Both the quasi-steady and fully unsteady approaches were implemented for this analysis, and their results were compared to those predicted by the Küssner function.

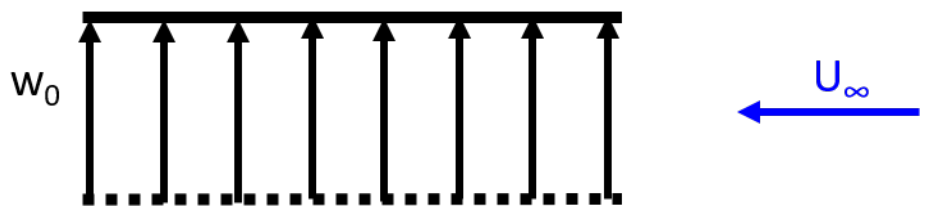

Figure 2.8: Sharp-edge gust model.

In order to simulate a two-dimensional case with this potential flow method, symmetry boundary conditions were applied at both ends of the wing (i.e. zero vorticity), resulting in a two-dimensional analysis.

A comparison of the quasi-steady and fully unsteady models against the Küssner function was first made, shown in Fig. 2.9. It is clear, the addition of the apparent-mass term makes a significant contribution, specifically in the early encountering of the gust, when the entire chord of the wing may not be influenced by the step increase in velocity. It is in this early time frame, also, that the largest changes in circulation, with respect to time, are seen; these changes are what are to be captured by the apparent mass term in Eq. 2.7).. The fully unsteady model shows good agreement with the analytical solution of the Küssner function. A slight over-prediction is seen in the early time steps, however, the steady state response matches very closely. The spike in lift coefficient in the fully unsteady results can be attributed to the time at which the leading edge of the gust reaches the trailing edge of the lifting surface.

The level of agreement with the analytical solution and sensitivity of the results using the unsteady model is highly dependent on the number of chordwise DVEs used. An increased number of chordwise elements provides an improved estimate of the chordwise circulation distribution. This distribution is especially important in gust analysis since different chordwise locations will experience different induced velocities and consequently a chordwise circulation gradient will be present. Figure 2.10 compares the results using the fully unsteady model with three different numbers of chordwise rows of elements, $m$. As expected, more chordwise elements provides a better prediction to the Küssner function. The spike 


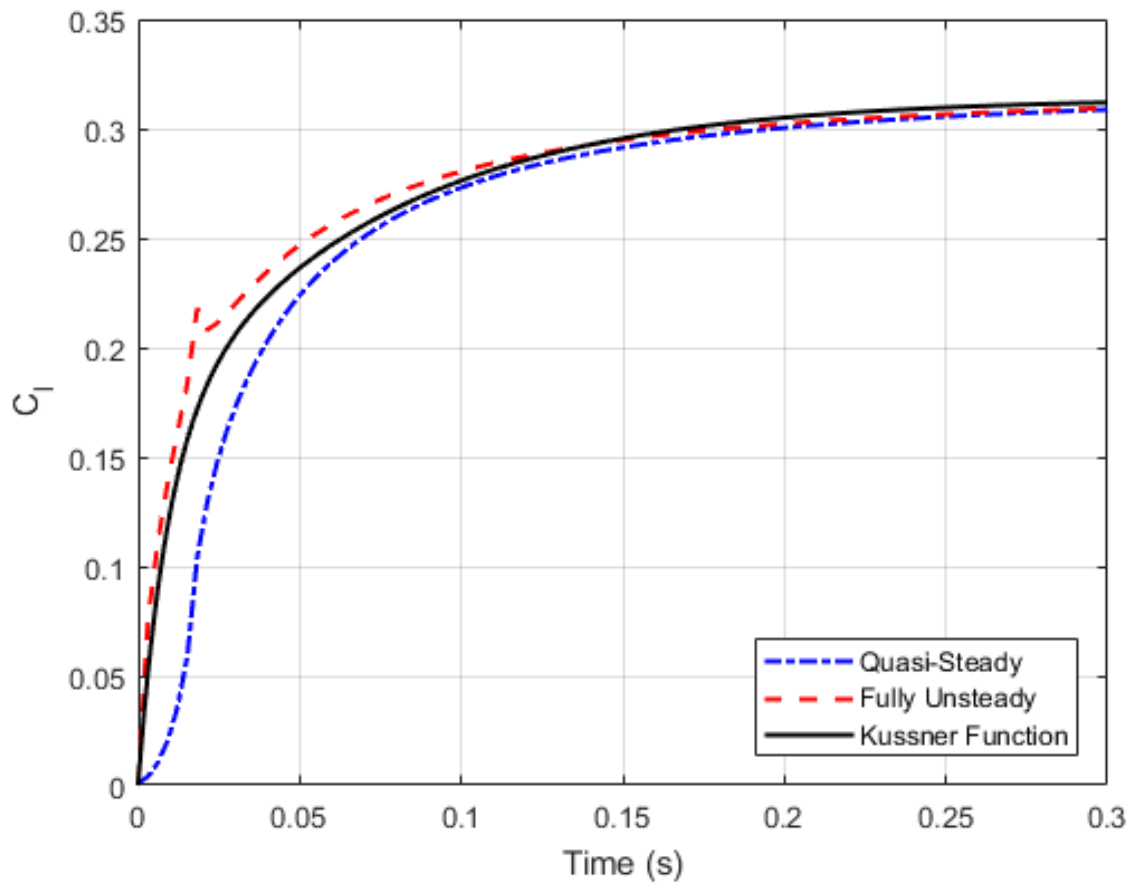

Figure 2.9: Comparison of quasi-steady and fully unsteady response to sharp-edge gust with 12 spanwise and 6 chordwise elements.

in lift coefficient when the gust reaches the trailing edge is also reduced with more elements.

\subsection{Summary}

A higher-order potential flow method was presented for use in a fluid-structure interaction model. The higher-order method provides comparable force resolution to more conventional approaches, however, with fewer elements. Induced drag predictions using the higher-order method are more numerically robust in comparison to approaches that are prone to singularity issues with vortex elements. A comparison was made between fixed and relaxed wake models in order to determine the merits of using each; the fixed wake model, while having reduced force resolution, provides significantly improved computation time when compared to a relaxed wake model. As a result, the fixed wake model was selected for future studies. A fully unsteady model was also shown for integration with the higher-order method; the fully unsteady model shows better agreement with analytical solutions in comparison to the existing quasi-steady approach and thus, is used for the remaining studies. Six chordwise elements were required to better resolve the transient lift response in comparison to analytical results. 


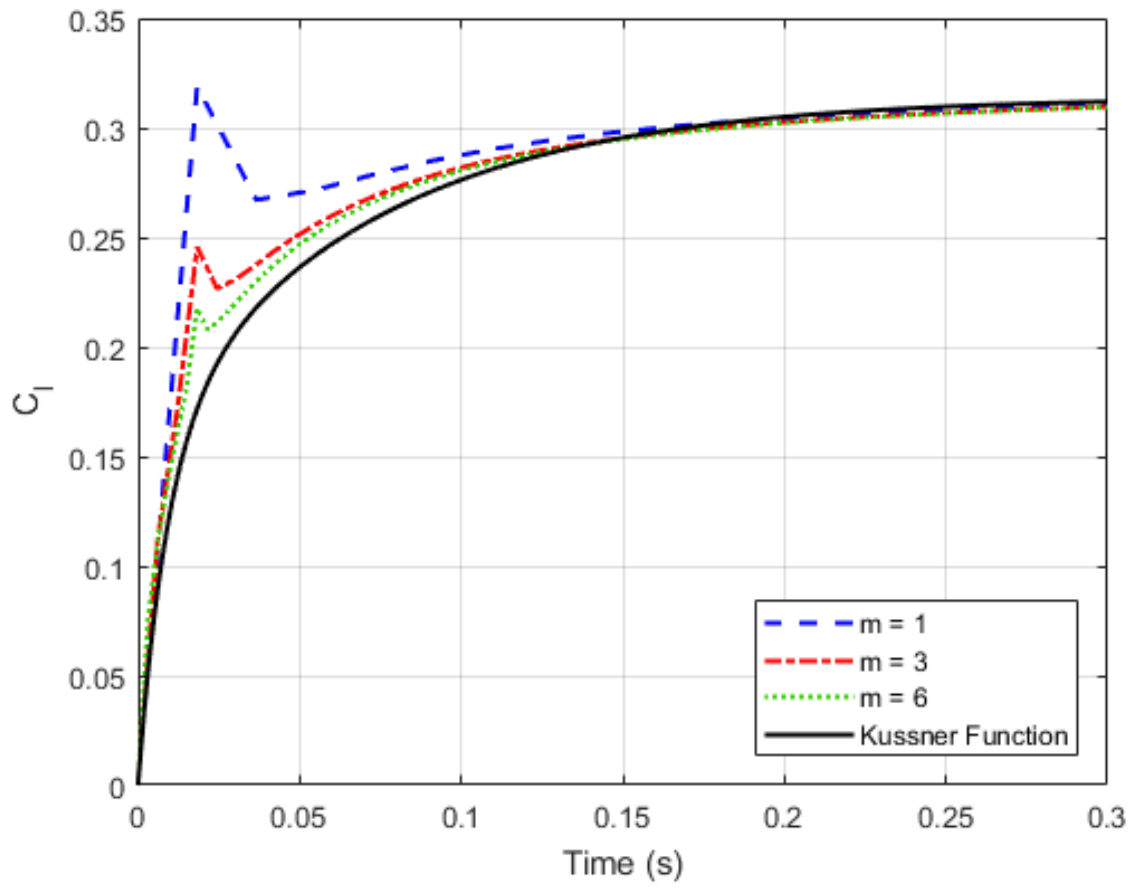

Figure 2.10: Validation of unsteady aerodynamics module with analytical solution to the Küssner function with varying chordwise elements. 


\section{Chapter 3}

\section{Finite Difference Structural Model}

To accurately model the aeroelastic behaviour of flexible wings, a structural dynamics model is coupled with the higher-order potential flow method described above. The structural dynamics is modelled using an explicit finite difference method to approximate the solution to the dynamic Euler-Bernoulli beam equations. Finite difference methods offer an efficient means to obtain accurate solutions to complex problems. In industry and academia it is also common to use Finite Element Methods (FEM). A disadvantage to using these approaches is, however, the need for extensive pre-processing work. A model must be constructed with the appropriate elements (beam, spring, shell, etc.), and a refined mesh must be created; with complex geometries, the meshing process can be tedious and require many iterations. At early design stages, one is interested in investigating a wide variety of configurations, thus performing the setup work required for an FEM approach multiple times is not ideal.

In comparison, finite difference methods require very little setup time; in terms of structural dynamics, beam properties may be specified as numerical functions and, consequently, can be easily modified from one configuration to the next. No meshing is required as properties are specified, based on these functions, at constant intervals across the length of the beam. These methods are typically more computationally efficient than FEM approaches; exact solutions to the governing equations can be found nearly instantaneously, and memory requirements, especially for explicit methods, are typically quite low. 


\subsection{Governing Equations}

The equations of motion for a dynamic system are a function of the mass and stiffness of the system. A simple spring and mass damper can be modelled through:

$$
\mathbf{M} \underline{\ddot{x}}+\mathbf{C}_{\mathbf{k}} \underline{\dot{x}}+\mathbf{K} \underline{x}=\underline{f}(x, t)
$$

where $M, C_{k}$ and $K$ are the mass, damping and stiffness matrices respectively. A time dependent force function, $f(x, t)$, may be applied in order to achieve a transient response. A commonly used representation of dynamic beam equations of motion is the Euler-Bernoulli beam equations. EulerBernoulli beam theory ignores deformations as a result of shear stresses, while Timoshenko beam theory includes this; assuming a slender structure (i.e. large thickness to length ratio) the error between the two models is small, however, shorter, thicker structures are better modelled through Timoshenko beam theory. The dynamic equations for Euler-Bernoulli and Timoshenko beams follow a similar form to that of (3.1), however, are specific to beam properties. The bending of a thin beam from a time dependent distributed load, using Euler-Bernoulli beam theory, can be described via [18]:

$$
\frac{\partial^{2}}{\partial x^{2}}\left(E I(x) \frac{\partial^{2} h}{\partial x^{2}}\right)=-\mu(x) \frac{\partial^{2} h}{\partial t^{2}}+f(x, t)
$$

where $E I$ is the bending stiffness, $\mu$ is the mass per unit length, $f$ is the distributed force and $h$ is the computed displacement. The torsional response to a distributed torque can be represented in a similar form:

$$
\frac{\partial}{\partial x}\left(G J(x) \frac{\partial \theta}{\partial x}\right)=\frac{\mu(x) I_{p}(x)}{A(x)} \frac{\partial^{2} \theta}{\partial t^{2}}+\tau(x, t)
$$

where $G J$ is the torsional stiffness, $I_{p}$ is the polar moment of inertia, $A$ is the local cross sectional area, $\tau$ is the distributed moment and $\theta$ is the computed twist response.

When applying these equations to an aircraft wing, a coupled form of the equations must be derived. The dynamic response of a wing to applied aerodynamic loads results in a coupled bending and twist response. Specifically from the dynamics, the coupling arises as a result of an offset between the shear and mass centers of the wing structure [3]. This offset is created due to the non-symmetrical nature of the wing structure. Simple structures with rectangular cross-sections, for example, will have shear and mass centers that coincide with one another, thus resulting in a decoupled response. Typically wing structures often conform very closely to the airfoil cross-section, which is not symmetric about any axis. Consequently, the offset between the shear and mass centers causes an inertial moment that creates a 
torsional acceleration, $\left(\frac{\partial^{2} \theta}{\partial t^{2}}\right)$, as the structure bends; a bending acceleration, $\left(\frac{\partial^{2} h}{\partial t^{2}}\right)$, and restoring inertial force is also created as a result of the torsion of the structure. To minimize the possibility of static aeroelastic instability, the shear center is placed near or in front of the aerodynamic center; this reduces the lift induced torque on the structure. Dynamic aeroelastic instabilities are mitigated by placing the mass axis in front of the elastic axis.

Based upon the principles of Eqs. (3.2) and (3.3), the coupled equations of motion for an aircraft wing may be derived. These equations are solved about the aeroelastic origin, which, for convenience, is placed at the shear center line. Figure 3.1 shows the coordinate system conventions. Aerodynamic lift and moment are resolved to the shear center as well as the inertial properties of the wing.

$$
\begin{gathered}
\mu(x)\left(\frac{\partial^{2} h}{\partial t^{2}}\right)-\mu(x) l_{s m} \frac{\partial^{2} \theta}{\partial t^{2}}+\frac{\partial^{2} E I}{\partial x^{2}} \frac{\partial^{2} h}{\partial x^{2}}+2 \frac{\partial E I}{\partial x} \frac{\partial^{3} h}{\partial x^{3}}+E I \frac{\partial^{4} h}{\partial x^{4}}=L^{\prime}(x, t) \\
\mu\left(l_{s m}^{2}+\frac{I_{p}(x)}{A(x)}\right) \frac{\partial^{2} \theta}{\partial t^{2}}-\mu(x) l_{s m} \frac{\partial^{2} h}{\partial t^{2}}-\frac{\partial G J}{\partial x} \frac{\partial \theta}{\partial x}+G J(x) \frac{\partial^{2} \theta}{\partial x^{2}}=M^{\prime}(x, t)
\end{gathered}
$$

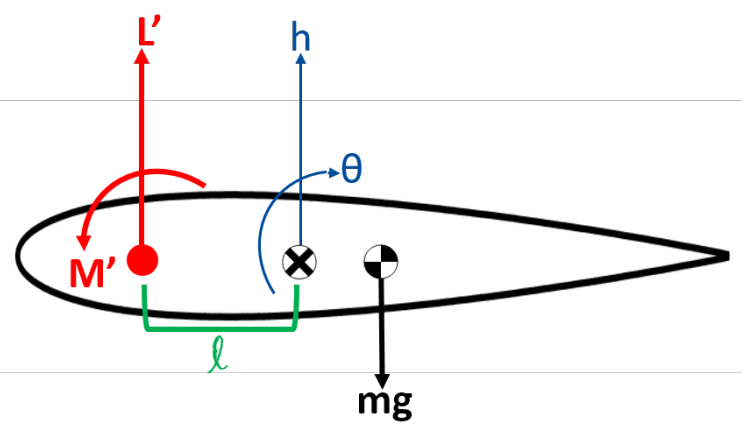

Figure 3.1: Coordinate system used for aeroelastic analysis.

Equations (3.4) and (3.5) are a system of partial differential equations that can be solved concurrently. In order to solve these equations, several boundary conditions must be defined. For the coupled bending dynamics described in Eq. (3.4), four boundary conditions must be defined to solve the fourth-order partial differential equation. An aircraft wing is often modelled as a cantilevered beam, thus, two boundary conditions must be applied at the root, namely, zero displacement and zero slope:

$$
\left.h\right|_{t, x=0}=\left.\frac{\partial h}{\partial x}\right|_{t, x=0}=0
$$

The remaining two boundary conditions are applied at the wing tip, specifying zero shear force and zero 
internal bending moment.

$$
\left.\frac{\partial^{2} h}{\partial x^{2}}\right|_{t, x=\frac{b}{2}}=\left.\frac{\partial^{3} h}{\partial x^{3}}\right|_{t, x=\frac{b}{2}}=0
$$

Since the coupled dynamic torsional equation is only a second-order partial differential equation, only two boundary conditions are required. At the wing root, a condition of zero twist is applied; at the wing tip, a zero slope boundary condition is applied.

$$
\left.\theta\right|_{t, x=0}=\left.\frac{\partial \theta}{\partial x}\right|_{t, x=\frac{b}{2}}=0
$$

\subsection{Explicit Finite Difference Method}

In order to solve the structural dynamic equations of motion, namely Eqs. (3.4) and (3.5), a form of numerical time and space integration is required. To accomplish this, an explicit finite difference method was used to approximate the partial derivative relationships in terms of simple fractional expressions. This method was used in a similar manner by Ironside et al. [19] to investigate the aeroelastic response of a high performance sailplane wing. The finite difference method discretizes the structure into several nodal grid points; at each of these grid points, the equations of motion are solved and a response of the entire wing is formulated. This is depicted in Fig. 3.2. the structural response is computed at each indicated nodal point, which are spaced by a constant amount, $\Delta y$, based on the applied aerodynamic load.

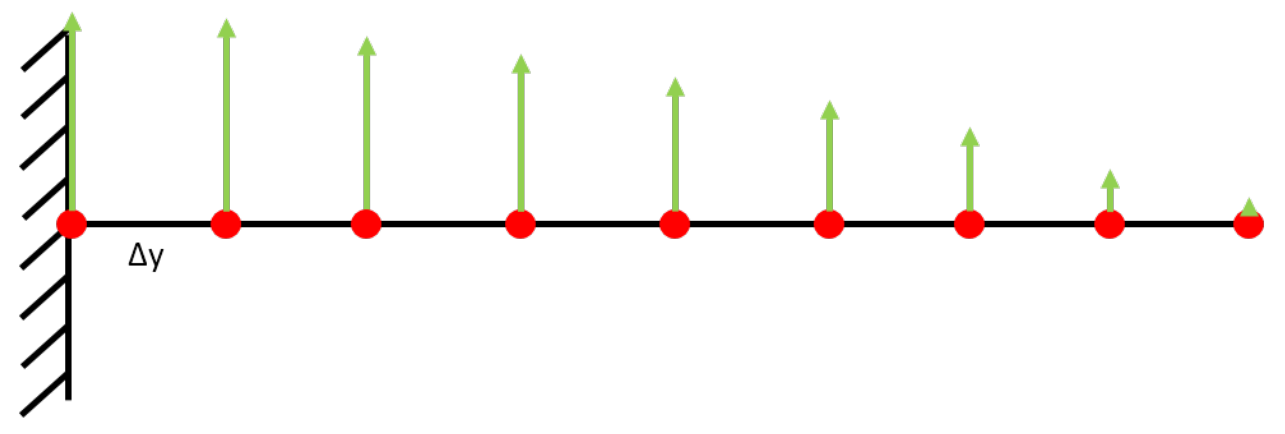

Figure 3.2: Discretization used for finite difference structural model.

An advantage to this approach is that an unequal number of aerodynamic and structural elements may be used; when this is the case, aerodynamic forces are interpolated at the structural points so that the finite difference relationships can be used. Since the aerodynamic model requires very few spanwise 
elements for accurate force resolution, a great number of structural elements is required, and thus, the above interpolation approach is employed for this research.

The finite difference relationships for the bending dynamics utilize a centered in space and forward in time discretization approach. Spatial derivatives at a given node point use solutions at surrounding grid points on the left and right to approximate the derivatives. Temporal derivatives use the solutions at current and previous time steps to determine the solution at a future time. The centered in space and time approach provides an estimation accuracy on the order of $O\left(\Delta x^{2}, \Delta t\right)$. Thus, the solution accuracy provided by the finite difference method is a function of both the grid spacing and time step size chosen. The relationships in Eqs. (3.9)- 3.12 are used to approximate the partial derivatives of Eq. (3.4).

$$
\begin{gathered}
\frac{\partial^{4} h}{\partial x^{4}}=\frac{h_{i+2}^{n}-4 h_{i+1}^{n}+6 h_{i}^{n}-4 h_{i-1}^{n}+h_{i-2}^{n}}{\Delta x^{4}} \\
\frac{\partial^{3} h}{\partial x^{3}}=\frac{h_{i+2}^{n}-3 h_{i+1}^{n}+3 h_{i}^{n}-h_{i-1}^{n}}{\Delta x^{3}} \\
\frac{\partial^{2} h}{\partial x^{2}}=\frac{h_{i+1}^{n}-2 h_{i}^{n}+h_{i-1}^{n}}{\Delta x^{2}} \\
\frac{\partial^{2} h}{\partial t^{2}}=\frac{h_{i}^{n+1}-2 h_{i}^{n}+h_{i}^{n-1}}{\Delta t^{2}}
\end{gathered}
$$

The derivatives in Eq. 3.5 are approximated in a similar fashion, shown in Eqs. (3.13)- 3.15).

$$
\begin{gathered}
\frac{\partial^{2} \theta}{\partial x^{2}}=\frac{\theta_{i+1}^{n}-2 \theta_{i}^{n}+\theta_{i-1}^{n}}{\Delta x^{2}} \\
\frac{\partial \theta}{\partial x}=\frac{\theta_{i+1}^{n}-\theta_{i-1}^{n}}{2 \Delta x} \\
\frac{\partial^{2} \theta}{\partial t^{2}}=\frac{\theta_{i}^{n+1}-2 \theta_{i}^{n}+\theta_{i}^{n-1}}{\Delta t^{2}}
\end{gathered}
$$

The expressions above utilize a subscript and exponent notation to denote spatial and temporal nodes. The subscripts, $i$, denote the spatial grid point at which the deflection or twist is used; the exponents, $n$, specify the temporal grid points to use. When solving the equations of motion, one needs to solve for the $n+1$ terms at each structural grid point, using the solutions at the previous two time steps, $n$ and $n-1$. The boundary conditions for the partial differential equations must also be implemented through 
finite difference relationships. The six boundary conditions form the following relationships:

$$
\begin{array}{rr}
h_{-1}^{n}=h_{1}^{n} & h_{N+2}^{n}=3 h_{N}^{n}-2 h_{N-1}^{n} \\
h_{0}^{n}=0 & \theta_{0}^{n}=0 \\
h_{N+1}^{n}=2 h_{N}^{n}-h_{N-1}^{n} & \theta_{N-1}^{n}=\theta_{N+1}^{n}
\end{array}
$$

where $N$ is the total number of structural node points across the wing.

The finite difference relationships that describe the coupled bending and torsional dynamics may be combined to form a single matrix equation in which the deflection and twist response of the structure is solved simultaneously. The matrix equation is provided in Ref. 19 but will be restated here.

$$
\left[\begin{array}{c}
h_{i}^{n+1} \\
\theta_{i}^{n+1}
\end{array}\right]=2\left[\begin{array}{c}
h_{i}^{n} \\
\theta_{i}^{n}
\end{array}\right]-\left[\begin{array}{c}
h_{i}^{n+1} \\
\theta_{i}^{n+1}
\end{array}\right]+\Delta t^{2} \mathbf{M}^{-1}\left(\left[\begin{array}{c}
f_{i}^{n} \\
\tau_{i}^{n}
\end{array}\right]-\mathbf{K}_{\mathbf{1}}\left[\begin{array}{c}
h_{x x} \\
0
\end{array}\right]-\mathbf{K}_{\mathbf{2}}\left[\begin{array}{c}
h_{x x x} \\
\theta_{x}
\end{array}\right]-\mathbf{K}_{\mathbf{3}}\left[\begin{array}{c}
h_{x x x x} \\
\theta_{x x}
\end{array}\right]\right)
$$

$M, K_{1}, K_{2}$ and $K_{3}$ represent that mass and stiffness matrices of the structure and $h_{x x}, \theta_{x}$ and similar terms are the finite difference expressions for partial derivatives with respect to $x$. The mass matrix contains the coupling terms that result in the simultaneous bending and torsional response. The main diagonal terms describe the mass distribution and torsional mass moment of inertia.

$$
\begin{gathered}
\mathbf{M}=\left[\begin{array}{cc}
\mu_{i} & -\mu_{i} l_{s m, i} \\
-\mu_{i} l_{s m, i} & \mu_{i}\left(l_{s m, i}^{2}-\frac{I_{p}}{A_{i}}\right)
\end{array}\right] \\
\mathbf{K}_{\mathbf{1}}=\left[\begin{array}{cc}
E I_{i}^{\prime \prime} & 0 \\
0 & 0
\end{array}\right] \quad \mathbf{K}_{\mathbf{2}}=\left[\begin{array}{cc}
E I_{i}^{\prime} & 0 \\
0 & -G J_{i}^{\prime}
\end{array}\right] \quad \mathbf{K}_{\mathbf{3}}=\left[\begin{array}{cc}
E I_{i} & 0 \\
0 & -G J_{i}
\end{array}\right]
\end{gathered}
$$

The stiffness matrices, $K_{1}$ and $K_{2}$, contain derivatives of both the bending and torsional stiffness. Since the finite difference method allows one to specify functions to represent the stiffness distributions, these derivatives do not need their own finite difference relationships. They can simply be the first and second order derivatives to the numerical functions. 


\subsection{Coupling Procedure}

The explicit finite difference method that is used in this study, discussed above, is tightly coupled with the higher-order potential flow method described in Chapter 2. The tightly coupled approach updates the aerodynamic and structural response at each time step, providing a good representation of the dynamics of an elastic wing. A tightly coupled approach is necessary when performing gust analysis, since the aerodynamics are constantly changing; at each time step a different load distribution is seen, which needs information to be constantly updated in the structural model. Loosely coupled approaches may not capture the entire dynamic behaviour and consequently provide a response that is not indicative of what truly would occur. An example of this is seen in Ref. 29, where a comparison was made between the transient responses of a tightly coupled and loosely coupled CFD/CSD approach. The responses in both bending and torsion of the tightly and loosely coupled approaches are shown in Fig. 3.3, represented by the solid and dashed lines respectively. The loosely coupled approach shows a significantly different response, predicting a lower frequency and amplitude in the torsional response; the bending response is not affected nearly as much, but does show an over-prediction in tip deflection over time. This result highlights the need for a tightly coupled model.
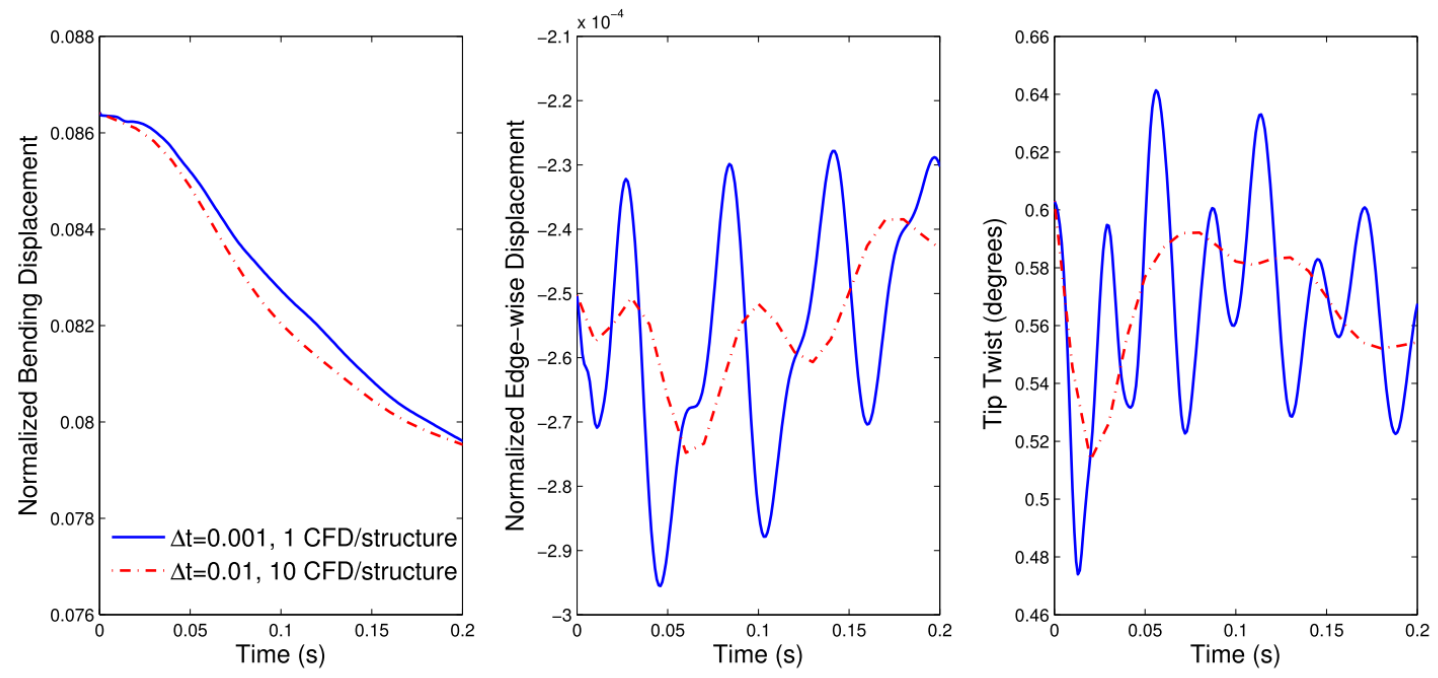

Figure 3.3: Comparison between tightly and loosely coupled approaches [29].

The coupling procedure requires that the induced velocities are computed at each time step after the structural response is determined. This ensures that the aerodynamics are captured as a result of elastic deformations. Due to the elastic deformation motion, the wing experiences what is known as aerodynamic damping. Aerodynamic damping occurs as a result of the increase and reduction in 
aerodynamic forces as the wing deforms up and down. The wing experiences a similar behaviour to that shown in Fig. 1.2 as the wing deflects upwards, a negative induced velocity is experienced, creating a reduciton in lift force. As the lift force is reduced, the structure begins to deflect downward, and a positive induced velocity is experienced. This creates an increases in the lift force and the cycle repeats itself; eventually the increase and decrease in lift is minimal and the wing reaches an equilibrium position. The addition of induced velocities as a result of elastic deformations creates what is known as aerodynamic stiffness. As the wing deflects upwards, a negative induced velocity is seen, reducing the overall lift on the wing, preventing it from deflecting as far. When the wing deflects downwards, a positive induced velocity is experienced, increasing the lift produced and thus slowing the downward motion. As a result, the wing acts stiffer than it actually is due to the induced effects, causing its response to dampen out to a steady-state condition.

Figure 3.4 outlines the coupling procedure for the aeroelastic model. At each time step aerodynamic forces are calculated using the higher-order method. These forces are then passed to the explicit finite difference method to compute the bending and torsional response after a time of $\Delta t$. Based on the computed response, the wing geometry is updated to reflect the changes. Induced velocities are computed from the updated geometry by using a simple first order spatial derivative approximation; as shown in Eq. 3.20 , the induced velocity at each DVE across the span is computed based on the current and previous locations of the DVE control points and the time step size used. The assumption made is that the velocity relationship is linear between time steps; this is not an ideal approximation, however, with a small enough time step size, the wing displacements between time steps are small and thus a linear approximation holds.

$$
\dot{\boldsymbol{X}}_{i n d}=\frac{\boldsymbol{X}_{i}^{n}-\boldsymbol{X}_{i}^{n-1}}{\Delta t}
$$

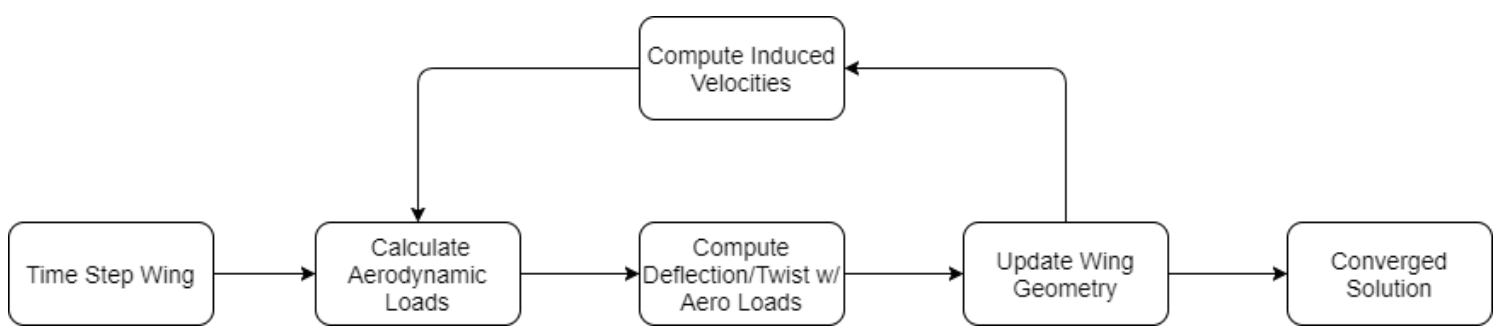

Figure 3.4: Coupling procedure for dynamic response of elastic wing. 


\subsection{Numerical Implementation}

The presented aeroelastic solver was implemented through the MATLAB programming environment. MATLAB provides a computationally efficient means of performing matrix operations through the use of a vectorized programming approach. The vectorized approach allows one to perform several numerical operations in a single step, without the need for conditional loops, which tend to be less efficient. For instance, the computation of wake induced velocities on a single element may be performed with one or two operations.

The programming algorithm follows a similar form to that shown in Fig. 3.4 . Two input files are required to perform an aeroelastic analysis; the first defines the aerodynamic surfaces, freestream conditions, analysis type and time stepping parameters, while the second defines the structural properties of the wing. The aerodynamic surface is defined through the use of panels, each with a specified number of spanwise and chordwise elements. Several panels may be used to model significant geometry changes such as the addition of sweep, taper or geometric twist to the wing. The structural input file defines the numerical properties for the dynamic solver as well as the bending and torsional stiffnesses, elastic and mass axis locations, and the mass distribution across the wing. These properties are defined through second order functions in the form of:

$$
f(x)=A x^{2}+B x+C
$$

thus only the coefficients of these functions are defined. Samples of these input files are provided in the appendix.

Based on the described input files, the lifting surface is discretized into the appropriate number of aerodynamic and structural elements. At each time step, the lifting surface is moved based on the defined freestream velocity and time step size. New wake elements are created as a result, and the circulation distribution on the surface is updated appropriately. For an aeroelastic analysis, the wing is moved for several time steps without computing any deflections in order to build up a wake and allow the initial aerodynamic forces to converge. Once the aerodynamic forces have converged, the algorithm shown in Fig. 3.4 is employed.

No defined output file is used for this solver; rather, all pertinent information such as the time history of deflections at each structural node and aerodynamic forces and coefficients at each time step are saved to the MATLAB workspace. This allows one to review the entire transient response as well as allowing for the program to be restarted from a specific time step. 


\section{Chapter 4}

\section{Model Validation}

\subsection{Static Aeroelastic Response}

A validation of the explicit method was first performed with a static aeroelasticity case. The static response of the Braunschweig SB-14 high performance sailplane, pictured in Fig. 4.1, was computed at two different flight speeds. The results from the explicit method were compared to those of a commercial Finite Element Model (FEM). The SB-14 aircraft was selected as a validation case since geometric and structural data were readily available. In addition, existing test results for multiple cases were made available, providing multiple validation points.

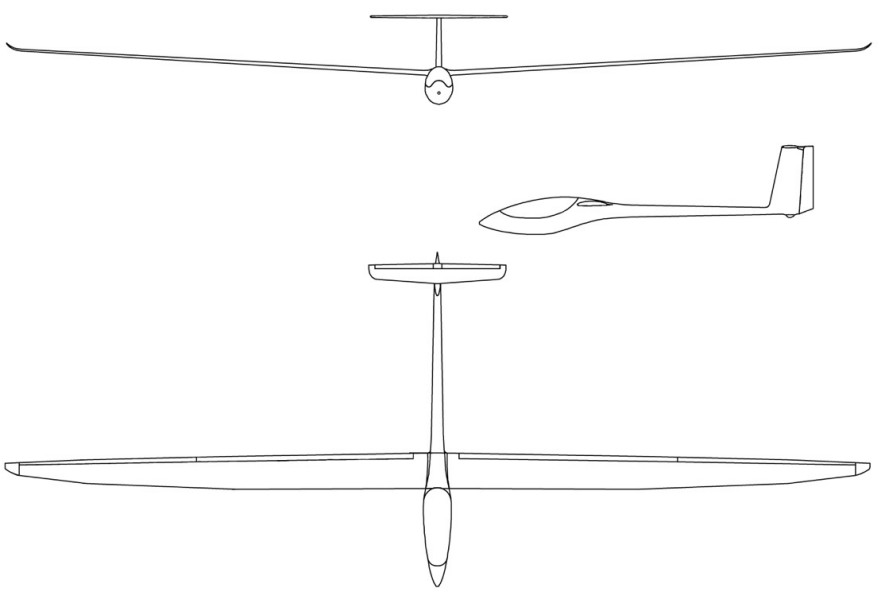

Figure 4.1: 3-view of Braunschweig SB-14 high performance sailplane [30]. 
The structural characteristics of the SB-14 were approximated with second-order functions; this includes the bending stiffness, torsional stiffness, linear mass density, and torsional mass moment of inertia. This approximation allows for an easy integration with the explicit method. The geometric properties of the aircraft are provided in Table 4.1. The lifting surface was modelled with 20 spanwise elements and 1 chordwise element; multiple chordwise elements were not required for this case since only a steady analysis was being performed. The change in force resolution for steady analysis with multiple chordwise elements is not enough to warrant the extra computation time required.

Table 4.1: Geometric characteristics for SB-14 aircraft.

\begin{tabular}{ll}
\hline \multicolumn{2}{c}{ Geometry } \\
\hline Wing Span $(\mathrm{m})$ & 18 \\
Planform Area $\left(\mathrm{m}^{2}\right)$ & 10.84 \\
Root Chord $(\mathrm{m})$ & 0.74 \\
Tip Chord $(\mathrm{m})$ & 0.259 \\
Aircraft Mass $(\mathrm{kg})$ & 350 \\
\hline
\end{tabular}

The first validation case was performed in steady level flight conditions, that is at 1 -g loads, at $30 \mathrm{~m} / \mathrm{s}$. The aerodynamic forces were allowed to reach convergence before being passed to the structural model. The computed static response is shown in Fig. 4.2. Both bending and torsional responses are compared against the commercial FEM results. The bending response in Fig. 4.2a shows good agreement with the commercial software, with minimal deviation. The torsional response, in Fig. 4.2b, is not predicted as well as bending; a consistent under-prediction is observed across the half-span. This difference is likely due to two factors. The torsional stiffness and mass moment of inertia is approximated as a second-order function rather than using the exact values. In addition, the explicit method models the wing structure as a cantilevered beam, while the FEM results model the entire aircraft; thus, there are wing-fuselage interactions that can impact the magnitude. Nevertheless, the mode shape is captured very well and the results provide confidence in the method moving forward.

An additional validation case study was performed at a higher flight speed of $60 \mathrm{~m} / \mathrm{s}$. This higher speed was chosen since it was expected that the torsional response is significantly different, as the pitching moment of the wing plays a more significant role. The results are summarized in Fig. 4.3 The bending response, again, shows good agreement with the FEM results. Only a slight deviation is noted near the wing tip. The torsional response is not predicted as well; an over-prediction is seen across most of the half-span, opposite to that seen in the $30 \mathrm{~m} / \mathrm{s}$ case. This again can be attributed to the approximation of structural properties, as well as the different boundary conditions applied at the wing root; the overall mode shape is still captured quite well. The under prediction at $30 \mathrm{~m} / \mathrm{s}$ and over prediction at $60 \mathrm{~m} / \mathrm{s}$ 


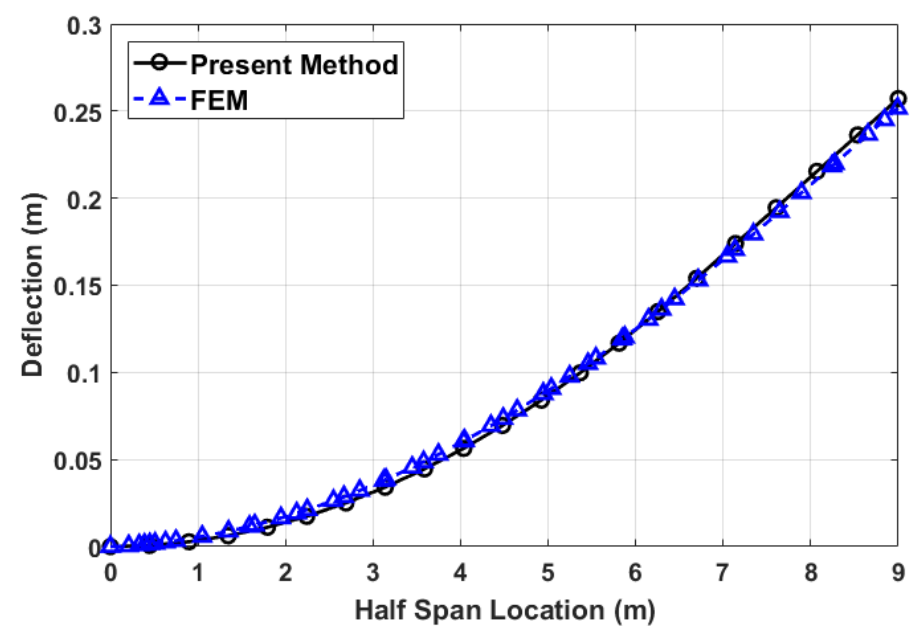

(a) Bending comparison

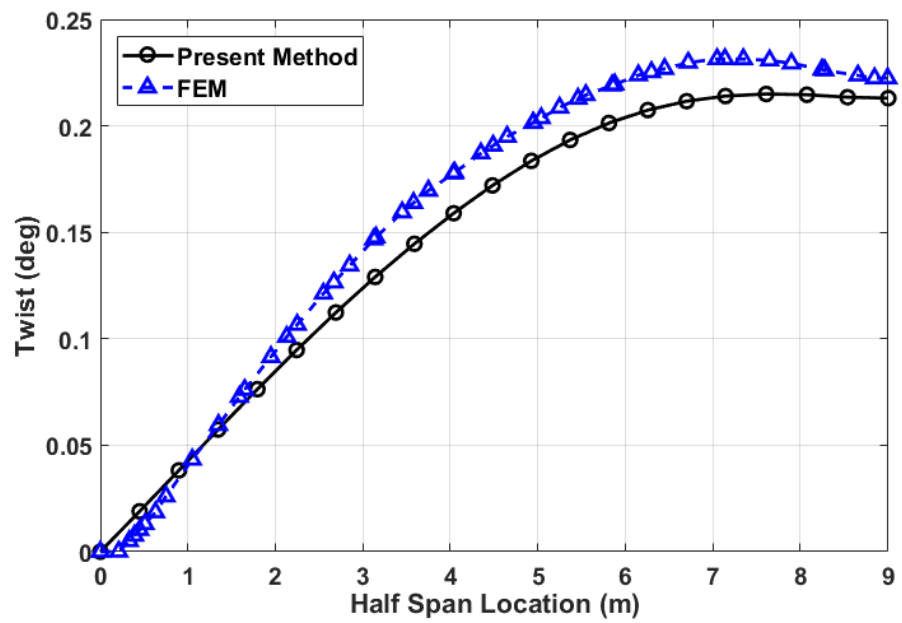

(b) Twist comparison

Figure 4.2: Static aeroelastic response of the SB14 at $30 \mathrm{~m} / \mathrm{s}$ using an explicit finite difference method.

could also mean that the aerodynamic pitching moment is being over predicted, resulting in a more pitch-down moment than seen in reality.

\subsection{Modal Analysis}

The dynamic structural response was also validated using a modal analysis to investigate the coupled natural frequencies of a cantilevered undergoing coupled bending and torsion. A similar analysis was performed by Banerjee [31] where an explicit frequency equation was developed to predict the natural 


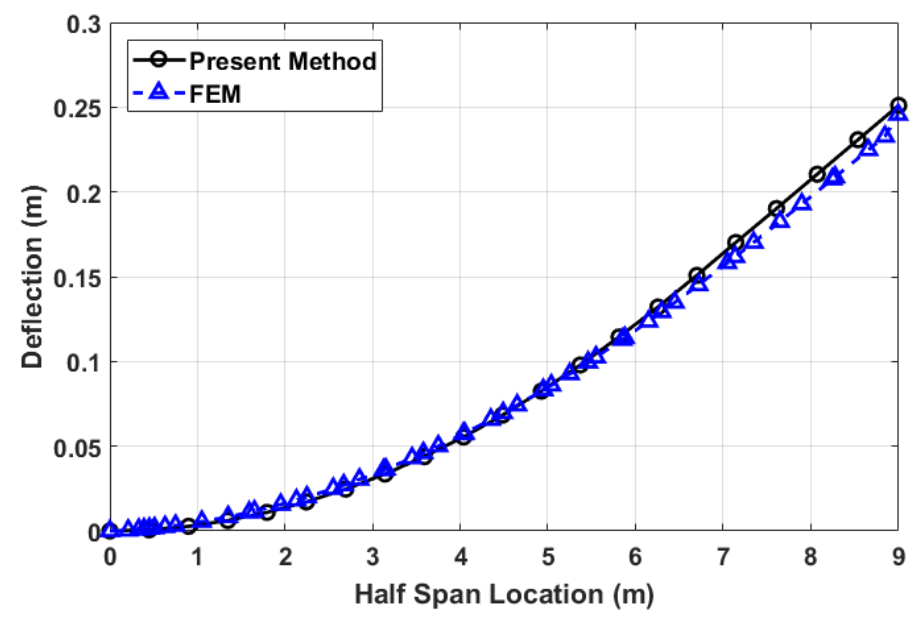

(a) Bending comparison

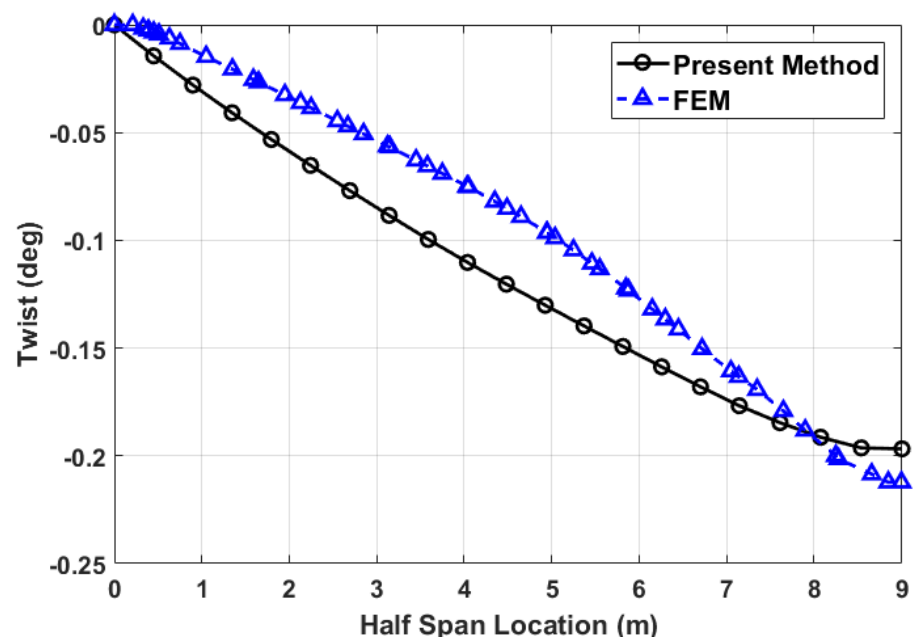

(b) Twist comparison

Figure 4.3: Static aeroelastic response of the SB14 at $60 \mathrm{~m} / \mathrm{s}$ using an explicit finite difference method.

frequencies of a coupled system. While the explicit finite difference method cannot directly perform a modal analysis, the time domain solution may be used in order to compute the frequency domain response. A case study was performed by Banerjee, where the developed explicit frequency prediction was used to predict the first two modal frequencies of the Goland wing. The same analysis was performed using the present finite difference method, and the results compared against those presented in Ref. 31 .

In order to obtain the frequency domain response, a fast Fourier transform (FFT) was used to convert the time domain results to the frequency domain and extract the dominant frequencies, as highlighted in Fig. 4.4. The results of the FFT show frequency on the x-axis and amplitude on the y-axis. The FFT 
was used to determine the dominant frequencies, which correspond to the major peaks in Fig. 4.4b

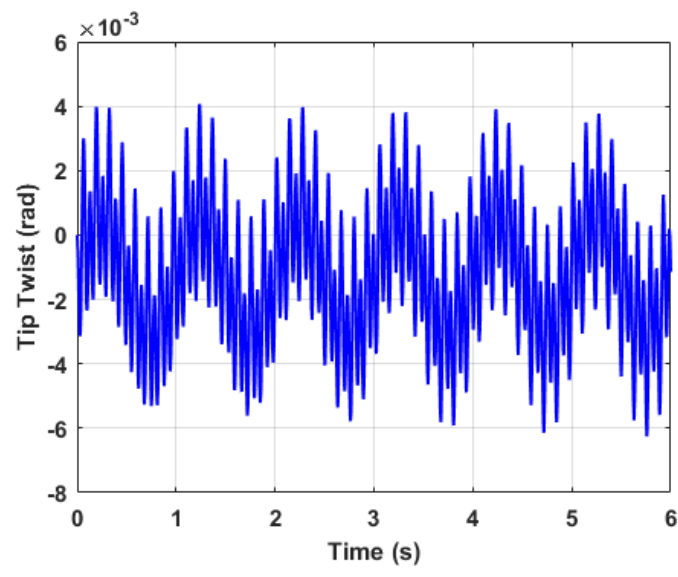

(a) Time domain response of tip twist.

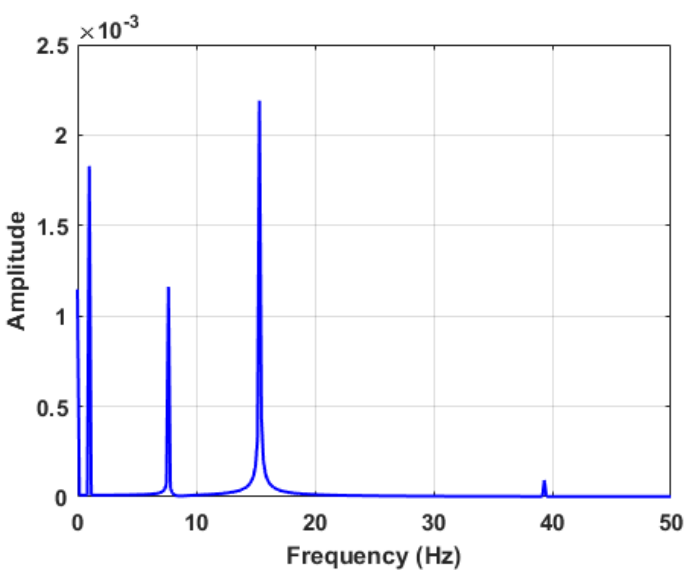

(b) Frequency domain response after performing FFT.

Figure 4.4: FFT analysis for free vibration of Goland wing.

To replicate the case study from Ref. 31, the present method was used to model the dynamic response of the Goland wing, which was subjected to a sinusoidal forcing function. The time domain response was recorded over a period of 6 seconds; an FFT analysis was then performed on the wing tip response in both deflection and twist. Three dominant frequencies were found, however, only two are of importance, since the first frequency corresponds to the frequency of the forcing function. For this analysis, a frequency of $1 \mathrm{~Hz}$ was used for the forcing function. The remaining two frequencies correspond to the natural frequencies of the first two modes of vibration. These frequencies were compared to those predicted by Banjeree and showed excellent agreement. A maximum relative error of 3.09\% was noted in predicting the first modal frequency, while the frequency of the second mode showed very little difference. These results provide confidence in the ability of the explicit finite difference method to accurately capture the dynamic structural response to an applied load.

Table 4.2: Comparison of coupled natural frequencies of Goland wing.

\begin{tabular}{cccc}
\hline Mode & Present Method (Hz) & Banerjee (Hz) & Relative Error (\%) \\
\hline 1 & 7.65 & 7.894 & 3.09 \\
2 & 15.37 & 15.438 & 0.44 \\
\hline
\end{tabular}




\subsection{Dynamic Aeroelastic Response}

The accuracy of the individual structural dynamic and aerodynamic models have been investigated. The coupling between the two, however, has yet to be explored. In order to validate an accurate coupling procedure, the effects of aerodynamic damping should be captured. To perform such an analysis, a rectangular wing with constant stiffness distributions in both bending and torsion was allowed to reach static aeroelastic convergence; convergence was deemed to have been met when the tip deflection and twist changed less than $2 \%$ between static iterations. Each iteration involved deforming the wing based on the aerodynamic loads; once deformed, new aerodynamic loads were computed and allowed to reach convergence. The process is then repeated until the convergence criteria were met. Induced velocities as a result of the elastic deformations were not considered since for a static analysis, one assumes that the wing moves from one state to another slow enough that these induced velocities are negligible. Once at static convergence, a sharp-edged gust with a length of $1 \mathrm{~m}$ and magnitude of $0.5 \mathrm{~m} / \mathrm{s}$ was introduced forcing the wing to deform.

Table 4.3 outlines the pertinent information for the wing configuration tested. The $32 \mathrm{~m}$ span was selected based on similar studies seen in Refs. 15 and 29, which represents a typical HALE aircraft configuration. The loading distribution for the rigid wing at the flight conditions specified in Table 4.3 is shown in Fig. 4.5 A NACA2412 airfoil was selected due to the well documented information available in literature; the airfoil also provides greater lift coefficients at low angles of attack when compared to common symmetrical airfoils, such as the NACA0012 used in Ref. 15. Thus, the loading distribution shown in Fig. 4.5 can be achieved at a variety of lift coefficients without the need to increase the angle of attack substantially. The structural properties were selected to ensure at the airspeeds that were considered for this analysis, elastic deformations remained in the range at which nonlinear effects are insignificant.

Table 4.3: Specifications for theoretical wing used for dynamic model validation.

\begin{tabular}{cc|cc|cc}
\hline \multicolumn{2}{c|}{ Geometry } & \multicolumn{2}{c|}{ Flight Conditions } & \multicolumn{2}{c}{ Structural Properties } \\
\hline Span $(\mathrm{m})$ & 32 & Airspeed $(\mathrm{m} / \mathrm{s})$ & 30 & Bending Stiffness $\left(\mathrm{N} \cdot \mathrm{m}^{2}\right)$ & 750,000 \\
Chord $(\mathrm{m})$ & 1 & Density $\left(\mathrm{kg} / \mathrm{m}^{3}\right)$ & 1.225 & Torsional Stiffness $\left(\mathrm{N} \cdot \mathrm{m}^{2}\right)$ & 750,000 \\
Airfoil & NACA2412 & Angle of Attack $(\mathrm{deg})$ & -1.25 & Linear Mass $(\mathrm{kg} / \mathrm{m})$ & 5.0 \\
& & & & Mass Moment of Inertia $(\mathrm{kg} \cdot \mathrm{m})$ & 2.0 \\
& & & Elastic Axis Location & $0.25 \mathrm{c}$ \\
& & & Mass Axis Location & $0.20 \mathrm{c}$ \\
\hline
\end{tabular}

The elastic axis was placed at the wing aerodynamic center to ensure unconditional static aeroelastic stability; the mass axis was placed further forward of here, towards the leading edge, to ensure uncondi- 
tional dynamic aeroelastic stability. An offset was specified between the elastic and mass axes to allow for the dynamic coupling between bending and torsion.

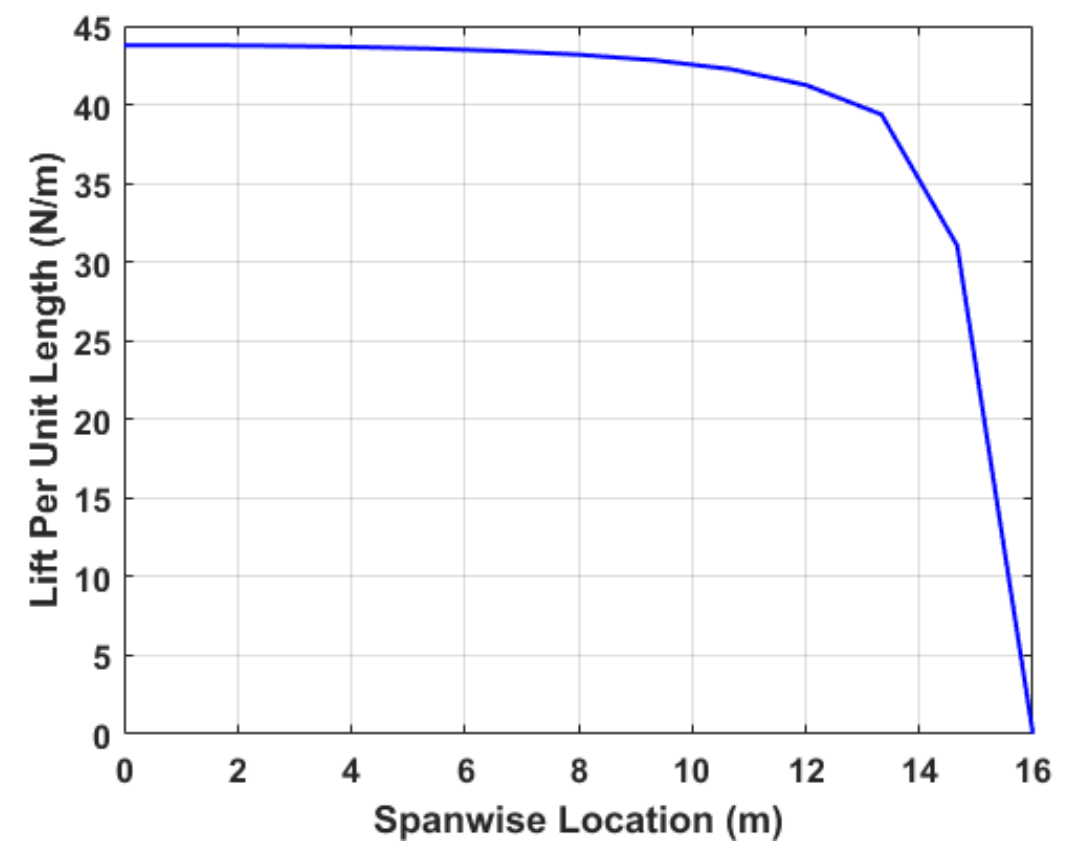

Figure 4.5: Spanwise loading distribution for test case wing geometry.

A panel density study was performed for the above geometry to determine the number of spanwise elements required for accurate force resolution. Figure 4.6 shows that between 12 and 15 spanwise elements, very little change in span efficiency prediction is seen. In the interest of improving computational time, 12 spanwise elements were chosen for all presented studies. Based on the results shown in Fig. 2.10. 6 chordwise elements were used for capturing the unsteady effects.

The dynamic bending and torsional response from the moment the wing encounters the gust to the point it returns to equilibrium was recorded and is shown in Fig. 4.7. One can see that the wing deviates from the equilibrium positions (denoted by the black dashed lines in Fig. 4.7) when encountering the gust; however, as the aerodynamic loads change as a result of the deformations, the wing is soon returned to the same equilibrium position. This is the expected result for a representative coupling procedure, as in absence of the gust, the wing returns to its previous equilibrium state. The coupled response in bending and torsion is also as expected; as the wing begins to deflect upwards, the tip experiences an increase in downward twist due to the inertial moment created from the mass axis being placed forward of the elastic axis. Changes in direction of the tip twist response correspond due inflection points in the tip deflection, thus, demonstrating their influence on one another. These results provide confidence that 


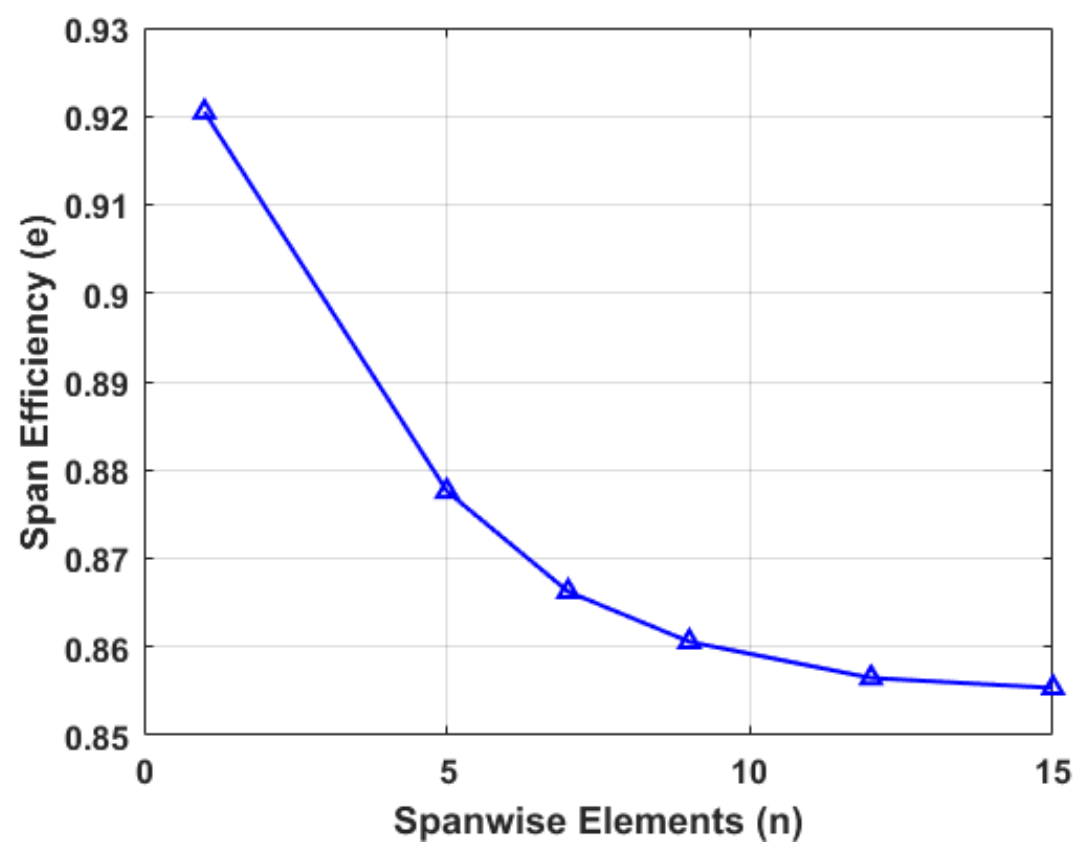

Figure 4.6: Sensitivity of span efficiency prediction to number of spanwise elements.

when coupled together, the aerodynamic and structural dynamic models capture the dynamic response of a wing encountering a gust in a satisfactory manner.

As a final note, the aerodynamic model required slight modifications in order to achieve a stable solution. The vortex filaments were removed from the wake as large induced velocities as a result of the filaments were experienced. This was due to the small time step size required for the structure model; this caused wake elements to be very small, resulting in filaments in close proximity to one another, thus creating the large induced velocities. The shed vorticity in the wake and apparent mass term on the lifting surface were still maintained, however, the absence of the filaments creates a violation of the Helmholtz theorem. This violation, however, does not pose a significant concern since with the small time step sizes, the step change in strength of the filament from one wake element to the next is not large enough to introduce significant error. 

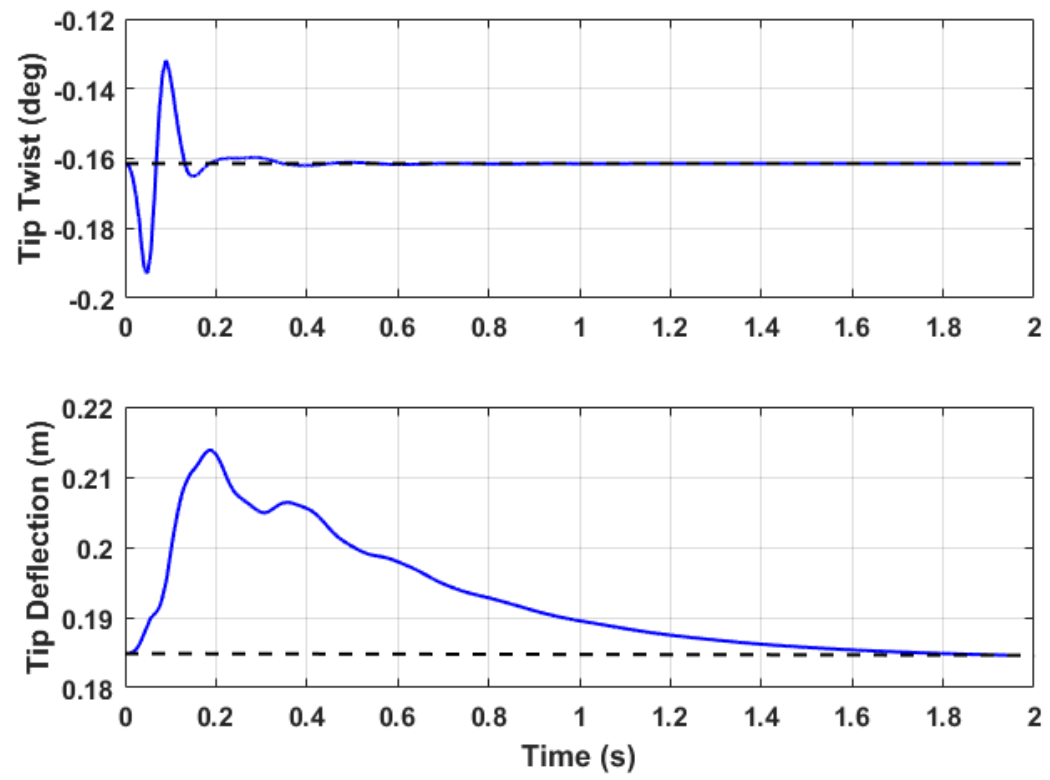

Figure 4.7: Effect of aerodynamic damping on dynamic structural response to sharp-edge gust. 


\section{Chapter 5}

\section{Gust Analysis}

Low-speed aircraft, such as UAVs and sailplanes, typically operate in a regime where atmospheric gusts with relatively small wave lengths, in terms of meters, are very prevalent. The strength and characteristics of these gusts are highly dependent on the atmospheric boundary layer, as discussed by Yeung [32]. The ground features play a significant role in affecting the magnitude and intensity of these gusts as they cause an increase in the size of the atmospheric boundary layer.

Aeroelastic gust analysis has been performed by several researchers, as discussed in Section 1 . Few, however, have investigated means for quantifying and improving gust performance. A means for quantifying the aerodynamic performance of an elastic wing encountering a gust will be described in this chapter.

\subsection{Gust Implementation}

The gust profiles are implemented in the same fashion described by Wang [25]. This method computes the magnitude of the gust velocity at a specific point based on its location relative to a reference point and the velocity at which the gust is traveling; such a gust profile is shown in Fig. 5.1. The gust velocities are applied at each time step to the DVE control points; based on the implementation method shown in Fig. 5.1. a wing with multiple chordwise elements will experience different velocities at different chordwise locations. The reference point, $x_{0}$, is chosen as the wing root leading edge, rather than the entire wing leading edge; thus, a swept wing may experience a velocity gradient across the span, depending on the gust speed and wing sweep. An example of this is shown in Fig. 5.2, which shows the implementation of an unsteady VLM [25]. The gust velocities are added to the control points, denoted by the red dots on 
the wing surface. Due to the tapered nature of this wing, creating a swept leading edge, and the straight gust profile, the wing root control points encounter the gust before the tip elements, thus creating a span wise velocity gradient. The same principles apply to the higher-order method used for this research.

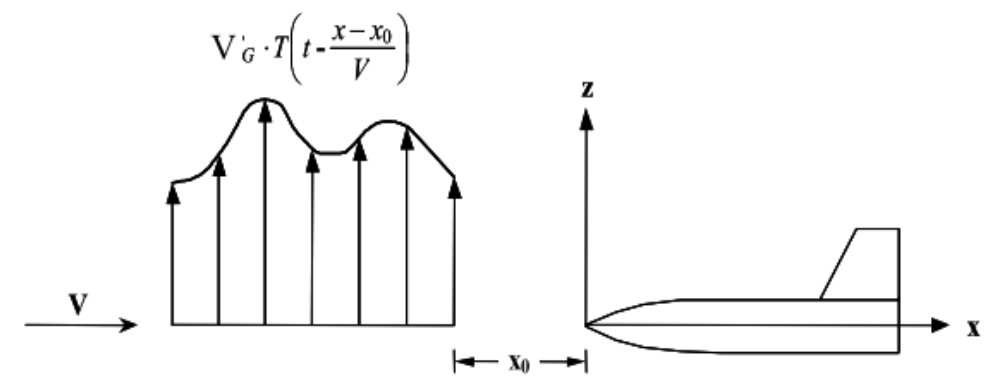

Figure 5.1: Implementation of a traveling gust with arbitrary profile [25].

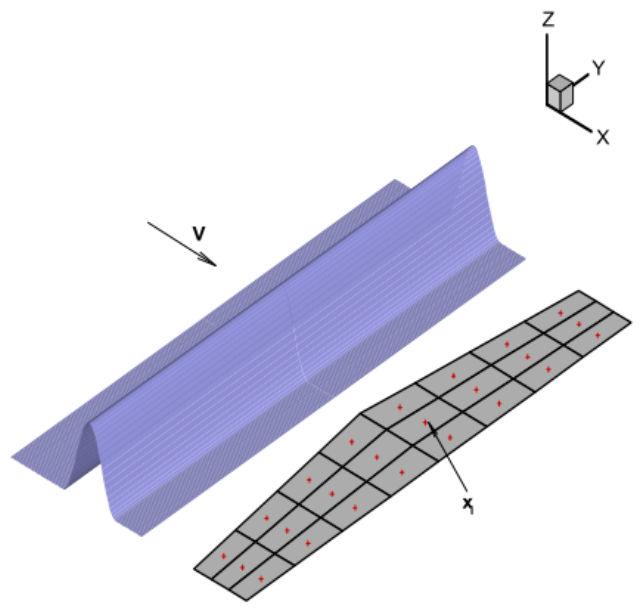

Figure 5.2: Incorporation of a gust profile with a VLM [25].

Two specific gust profiles were of interest for this research, namely the sharp-edge gust and 1-cosine gust, as shown in Fig. 5.3. While these profiles are not entirely representative of what occurs in practice, they are commonly accepted profiles for use in gust analysis, as exhibited in Refs. 7, 10, 16, and 33,

In the time domain, a 1-cosine gust can be represented by the following [25]:

$$
V_{G}(x, t)= \begin{cases}\frac{1}{2} w_{0}\left(1-\cos \frac{2 \pi \tau}{L_{G} / U_{\infty}}\right) & 0 \leq \tau \leq L_{G} / U_{\infty} \\ 0 & \tau \geq L_{G} / U_{\infty} \text { or } \tau<0\end{cases}
$$



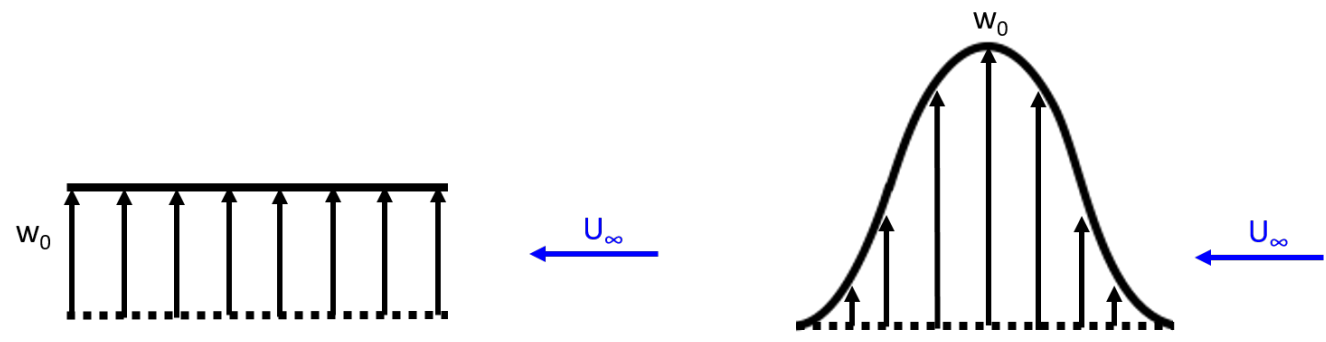

Figure 5.3: Two gust profiles investigated in case studies.

where $\tau$ is a time parameter that represents the amount of time that has passed since the point of interest has passed the reference point of the gust, $L_{G}$ is the wave length of the gust, $w_{0}$ the magnitude of the gust and $L_{G} / U_{\infty}$ is the period of the gust. The time parameter, $\tau$, can be described by the following:

$$
\tau=t-\frac{x-x_{0}}{U_{\infty}}
$$

The sharp-edge gust can be represented in a similar manner, with $\tau$ dictating when to apply the step-wise function.

$$
V_{G}(x, t)= \begin{cases}w_{0} & 0 \leq \tau \leq L_{G} / U_{\infty} \\ 0 & \tau \geq L_{G} / U_{\infty} \text { or } \tau<0\end{cases}
$$

The performance of an elastic wing encountering a gust is difficult to quantify as several performance parameters are constantly changing over time. Which performance metrics to focus attention on is not entirely intuitive depending on the goal of the analysis. In terms of energy extractions, changes in the drag acting on the wing are of primary importance. As a result of the tilting of the lift vector due to the gust, drag can be decreased; maximizing the magnitude at which the drag is decreased, is the goal of the energy extraction. Profile drag changes as a result of elastic deformations are assumed to be minimal, given small reduced frequencies, in comparison to induced drag changes; thus, the changes in induced drag should dominate the energy extraction.

Figure 5.4 shows a comparison between the induced and wing profile drag changes of an elastic wing encountering a 1-cosine gust. The same wing as outlined in Table 4.3 was used for this study, at an airspeed of $50 \mathrm{~m} / \mathrm{s}$. Maximum changes in wing profile drag of approximately $5 \%$ are seen, while changes in induced drag are nearly two orders of magnitude of the steady-state induced drag. This clearly shows that the changes in induced drag is the dominant term, thus allowing the use of potential flow methods 
for this analysis, which are computationally less demanding than higher fidelity methods such as a CFD based approach.

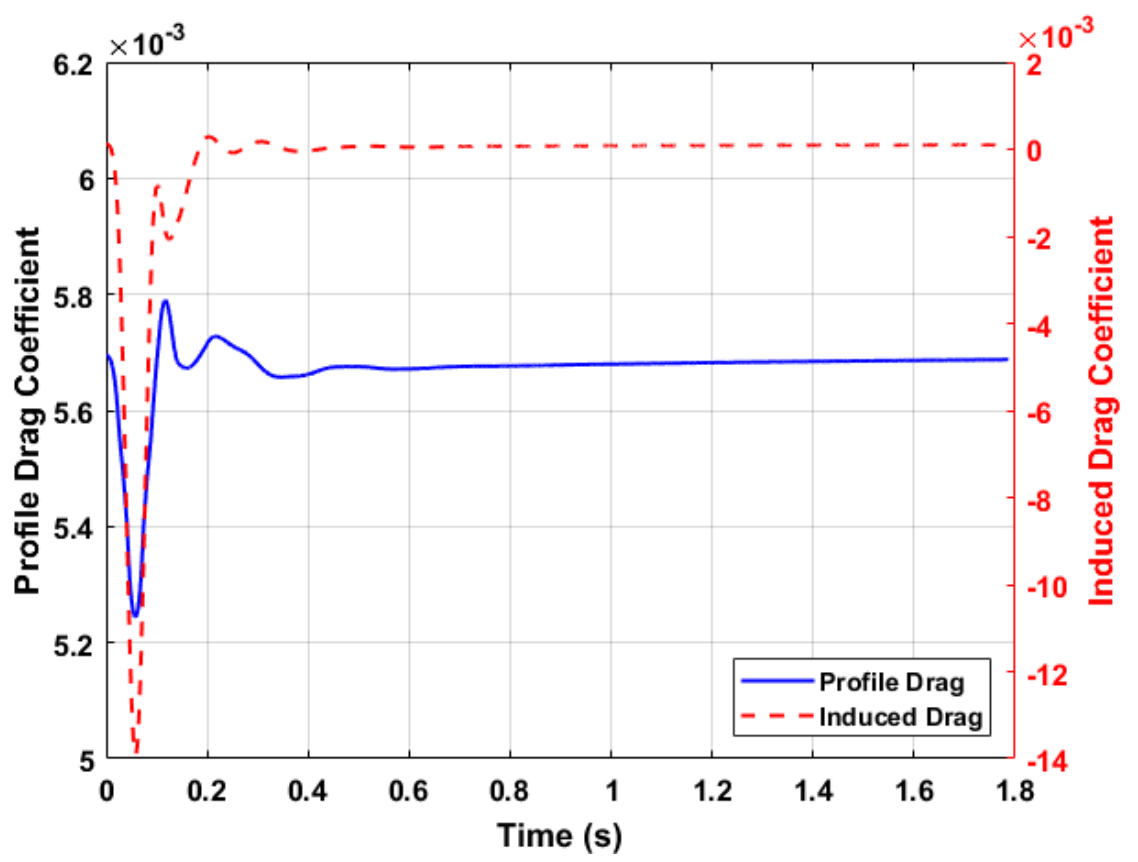

Figure 5.4: Changes to profile and induced drag as a result of traversing through a gust.

To provide a means to compare the drag changes from one configuration to the next, a term known as gust efficiency is defined. This term computes the average drag over the period of the gust profile, comparing it to the drag of the wing just before encountering the gust, as shown in Eq. (5.4) [34]:

$$
\eta_{\text {gust }}=-\frac{1}{T_{0}} \int_{0}^{T_{0}} \frac{\left(C_{D}\right)-\left(C_{D}\right)_{\text {ref }}}{\left(C_{D}\right)_{\text {ref }}} d t
$$

where $T_{0}$ is the time until the wing reaches its equilibrium state and $C_{D_{r e f}}$ is the drag coefficient of the wing just before encountering the gust. The efficiency can be thought of as how effectively the elastic wing is able to extract energy from the gust in comparison to the steady state configuration. A positive efficiency shows that energy has been harvested from the gust, while a negative efficiency indicates that additional work is required from the vehicle to traverse through the gust. 


\subsection{Application of Aeroelastic Tailoring}

A study of the effects of aeroelastic tailoring on the energy extraction capabilities of an elastic wing was performed using the same wing outlined in Table 4.3 Between each of the cases, the elastic axis location was allowed to vary and the gust efficiency of each configuration was recorded. While it was assumed for each case investigated that the desired elastic axis position and orientation could theoretically be achieved, it is important to consider how such a task could be accomplished in practice. The methods outlined by Francois et al. [14] present some unique techniques for shifting the elastic axis by changing the rib and spar orientations throughout the wing span. Additional measures such as shifting the spar locations along the chord, and modifying the size and shape of the wing box structure could be done in order to shift the elastic axis.

For each case, the wing was allowed to reach static aeroelastic convergence; this was defined as when the tip deflection and twist changed less than $2 \%$ based on the steady aerodynamic loads. Upon reaching convergence, the deformed wing was subjected to a 1-cosine gust profile; the gust had a magnitude of 4 $\mathrm{m} / \mathrm{s}$ and a wave length of $6 \mathrm{~m}$. The freestream velocity was prescribed to $50 \mathrm{~m} / \mathrm{s}$ in order to decrease the reduced frequency of the gust profile; based on the given conditions, the reduced frequency was 0.52 .

The first set of test cases investigated moving the entire elastic axis to different chordwise locations (i.e. having a constant elastic axis distribution). This, while not an entirely realistic possibility, aids in providing trends in elastic axis locations that maximize energy extractions. In addition, these cases provide a baseline for how much of an improvement (or lack there of), one can expect with a similar configuration.

Figure 5.5 shows the resulting gust efficiencies from moving the elastic axis from a chordwise location of $0.5 c$, forward to a location of $0.2 c$. Cases with the elastic axis between $0.3 c$ and $0.5 c$ represent configurations that could be subject to static instabilities such as aileron reversal or divergence, or at the very least, a loss in aileron effectiveness. With the mass axis always placed ahead of the elastic axis, however, the system is always dynamically stable. All cases showed significant improvements in energy extraction capabilities; an average wing drag reduction of $7.5 \%$ is seen at each elastic axis location. Moving the elastic axis towards the aerodynamic center showed slight improvements over configurations with the elastic axis further aft; between $0.4 c$ and $0.5 c$ an improvement of an additional $0.05 \%$ is seen. However, moving the elastic axis in front of the aerodynamic center to $0.3 c$ showed a reduction in the energy extracted in comparison to the previous two configurations. Similar research performed by Mai concluded that the majority of energy extractions from encountering a gust are more a result of the bending deformations than torsional deformation [7]. Thus, larger deformations when encountering a 
gust should result in a greater energy extraction. While the increase in downward twist as a result of the elastic axis being close to or in front of the aerodynamic center is beneficial for thrust generation, an increase in downward twist results in a lift decrease that reduces the bending deformations. At cases of $0.2 c$ and $0.3 c$, the bending deformations are significantly reduced from configurations with the elastic axis further aft. As a result, the wing begins to approach a behaviour similar to the rigid wing case. For these cases it appears that the benefits of a pitch down twist response are outweighed by the reduction in bending deformation, resulting in reduced drag reductions.

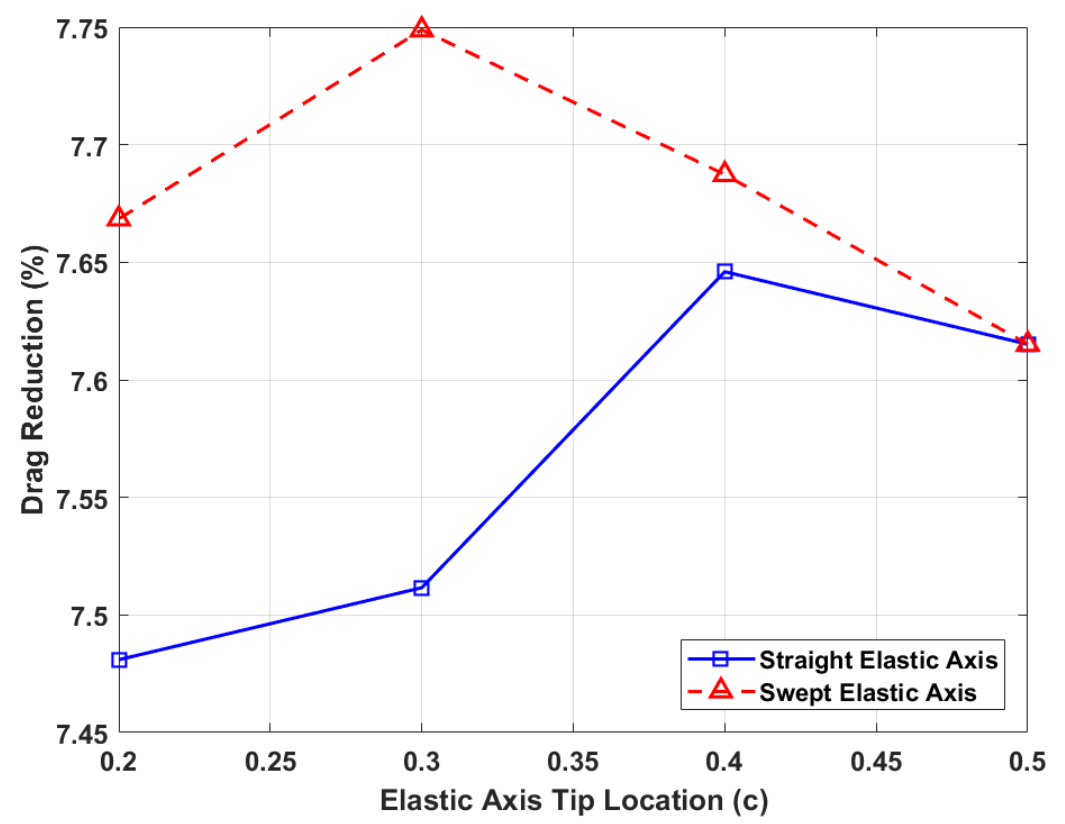

Figure 5.5: Drag reduction with varying chordwise location and orientation of the elastic axis.

The second case study investigated the effects of adding sweep to the elastic axis on the gust energy extraction capabilities. For this study, a linear variation in the elastic axis location was investigated. For each case, the root location was held fixed at $0.5 c$; the tip location of the elastic axis was allowed to vary between $0.5 c$ and $0.2 c$, thus adding between 0 and 1 degree of sweep. These results are also shown in Fig. 5.5. together with the previous unswept elastic axis results. The results for the swept configurations, indicated by the dashed lines, are plotted versus the tip location of the elastic axis for each case. The addition of sweep to the elastic axis provided a noticeable improvement over the results shown for the straight elastic axis configurations. At the very least, an improvement of approximately $0.05 \%$ was noted, while at the extreme end, a $0.25 \%$ improvement was seen. A swept elastic axis represents a configuration that, in practice, is much more easily achieved than shifting the entire elastic axis of the wing forward. 
Sweep can be achieved through tapering of the internal wing structure, coupled with careful orientation of composite layers. The results shown agree with trends found by Mai in that the shifting forward of the elastic axis demonstrate improved energy gains [7]. Increasing the forward sweep of the elastic axis increases the downward twist of the wing, thus reducing the lift increase from the gust; the forward tilting of the lift vector, thus, has a more pronounced effect in thrust production than would with large increases in lift. Since the entire elastic axis was not shifted forward, the negative effects of reduced tip deflection as a result of the increased pitch down response are not as noticeable. The reduction in energy extracted is not seen until a tip location of $0.2 c$, which is $10 \%$ later than the unswept elastic axis cases.

A more in-depth look into the transient response of the $0.3 c$ swept elastic axis case was performed to identify where the bulk of the wing drag reductions arise. A time history of the elastic response of the wing is shown in Fig. 5.6, along with the instantaneous value of the integrand from Eq. 5.4. This instantaneous value can be thought of as the instantaneous wing drag reduction at any given time. The results are plotted from the moment the leading edge of the wing encounters the gust; the instance the gust leaves the trailing edge of the wing is marked by the dashed lines. Since the gust velocity experienced across the chord varies, only the gust velocity of experienced by the leading edge elements over time is plotted. It is clear, the bulk of the drag reduction is seen in the first encountering of the gust. This corresponds to the pitch down torsional response and the initial increase in tip deflection. As the wing twist begins to reduce, the reduction in drag becomes less, however, the increase in tip deflection ensures that a drag increase is not seen; this result follows the trends outlined in Ref. 7. The residual response of the wing returning to its equilibrium state does not play as significant of a role in drag reductions, as evidenced by the near zero reductions shown past 0.2 seconds.

The same wing configuration was analyzed under rigid conditions to compare energy extraction capabilities. The rigid wing case showed very minimal drag reductions $(<1 \%)$, demonstrating that elastic deformations have a significant impact on the energy harvesting abilities. An additional 7.5\% reduction in drag is seen with a flexible wing, thus, it is necessary to include the effects of elastic deformations when determining the aerodynamic performance of wings traversing through gusts.

The effect of increasing the lift coefficient of the wing on the energy harvesting capabilities was also investigated. This was done using the case which showed the best drag reductions in Fig. 5.5, namely, a swept elastic axis with a tip location of $0.3 c$. While the wing drag reductions at sea level and cruise conditions show promising results, it is important to consider other flight conditions in the aircraft's operating range such as closer to its maximum aerodynamic efficiency operating point. The presented results in Fig. 5.5 were for a lift coefficient of approximately 0.09. Two other lift coefficients of 0.4 and 0.8 were also investigated. At each of these lift coefficients, the wing loading remained the same as 

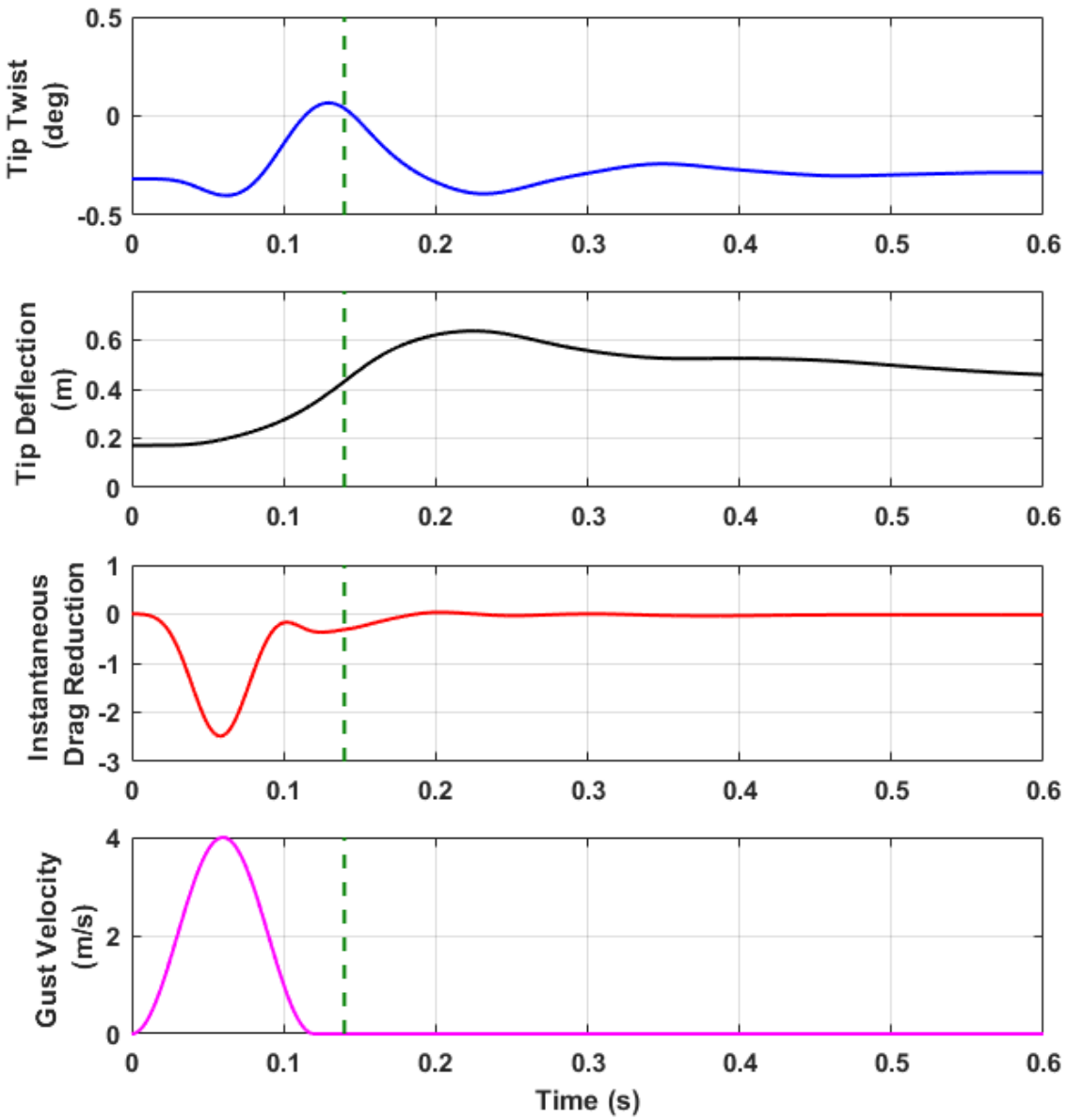

Figure 5.6: Time history of elastic response and instantaneous drag reduction for $0.3 \mathrm{c}$ swept elastic axis configuration.

previous cases as well as the freestream velocity and gust profile; thus, the reduced frequency remained unchanged. The freestream density was adjusted to keep the loading the same, essentially changing the altitude at which the aircraft is flying. Figure 5.7 shows the wing drag reduction as a function of the wing lift coefficient.

The drag reduction is improved to nearly $10.5 \%$ as the lift coefficient is increased to 0.4 , however, further increases cause a reduction in the energy harvested. This reduction is likely a result of the reduced elastic deformation changes at higher lift coefficients. Figure 5.8 shows a decomposition of the force coefficients, elastic deformations and instantaneous wing drag reductions at lift coefficients of both 0.4 and 0.8 , indicated by the dashed and solid lines respectively. At a $C_{L}$ of 0.8 , when encountering the 


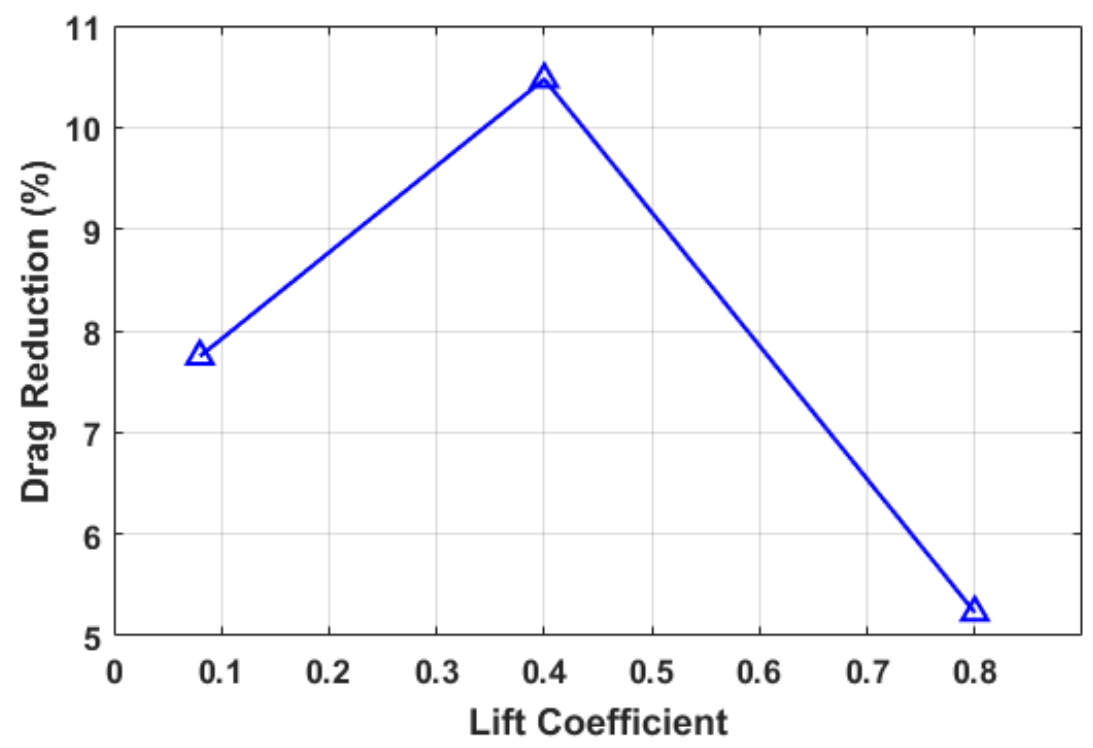

Figure 5.7: Dependency of wing drag reduction on lift coefficient.

gust, the tip deflection and twist deviate less from their equilibrium position than at a $C_{L}$ of 0.4 . As a result, the benefits of increased tip deflections in the energy extraction process are reduced. This is evident when investigating the induced drag response in Fig. 5.8. At a $C_{L}$ of 0.4 , the induced drag is reduced by a factor of nearly 8 , while at a $C_{L}$ of 0.8 , the induced drag is only reduced by a factor of approximately 3 from its steady-state value. Since profile drag changes are minimal, this results in a reduced energy extraction. These results show that the lift coefficient plays a large role in the energy extraction process, and further design exploration is required for tailoring the wing structure at higher lift coefficients.

Another gust profile that was studied is a sinusoidal gust, which involves both a positive and negative vertical gust velocity, and thus, results in a net zero air mass motion. The sinusoidal profile was implemented in a piece-wise fashion, combining 1-cosine and cosine wave forms. This was done to allow for a slower build-up and drop off of the gust velocity, rather than a sharp increase and decrease, which would normally be seen with a typical sine wave.

$$
V_{G}(x, t)= \begin{cases}\frac{1}{2} w_{0}\left(1-\cos \frac{2 \pi \tau}{0.5 L_{G} / U_{\infty}}\right) & 0 \leq \tau \leq 0.25 L_{G} / U_{\infty} \\ -w_{0}\left(\cos \frac{2 \pi \tau}{L_{G} / U_{\infty}}+\frac{3 \pi}{2 L_{G} / U_{\infty}}\right) & 0.25 L_{G} / U_{\infty}<\tau \leq 0.75 L_{G} / U_{\infty} \\ -\frac{1}{2} w_{0}\left(1-\cos \frac{2 \pi \tau}{0.5 L_{G} / U_{\infty}}\right) & 0.75 L_{G} / U_{\infty}<\tau \leq L_{G} / U_{\infty}\end{cases}
$$



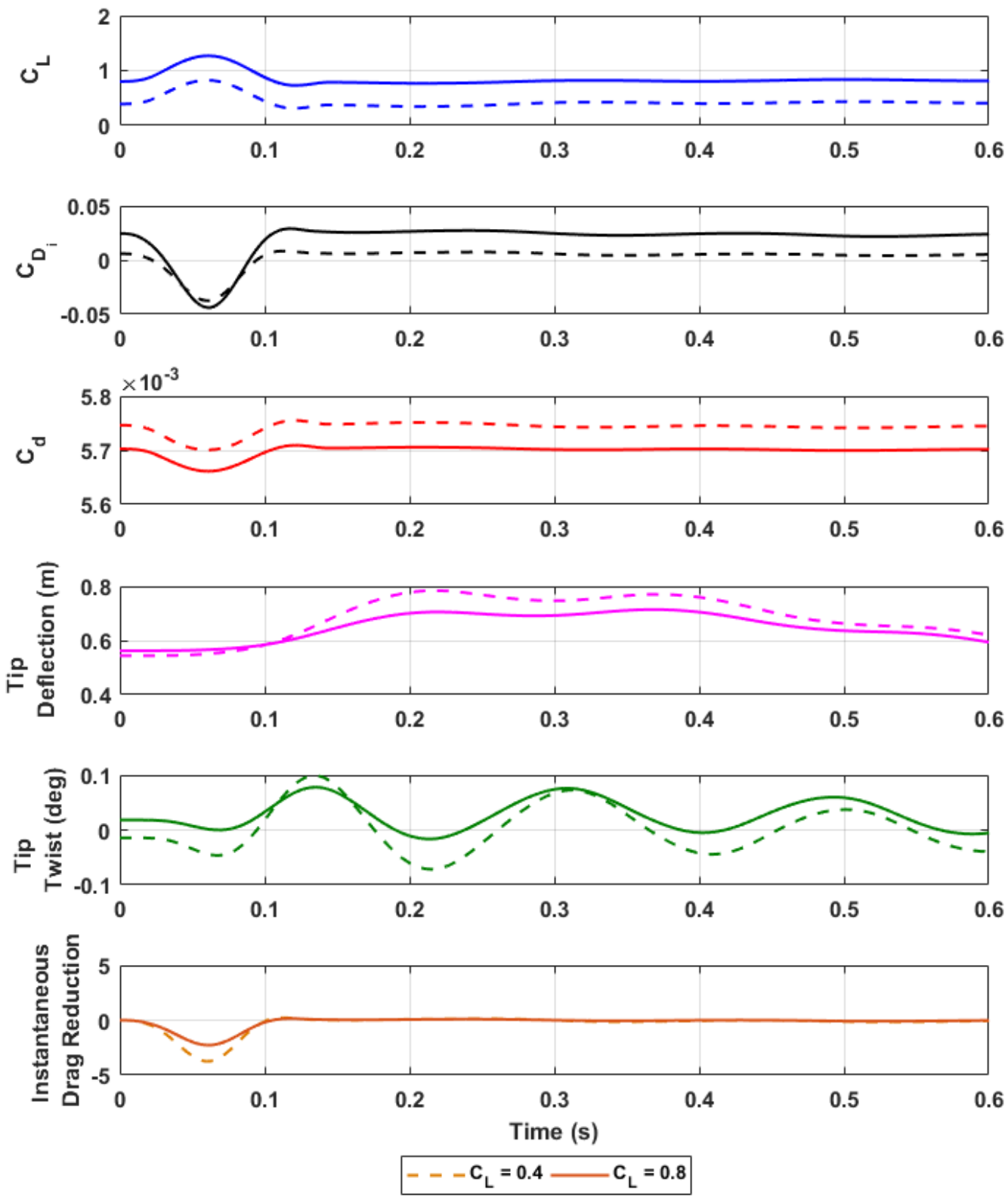

Figure 5.8: Time history of force coefficients and elastic deformations at lift coefficients of 0.4 (dashed) and 0.8 (solid).

Using the swept elastic axis with a tip location of $0.3 c$, at a lift coefficient of 0.4 , a sinusoidal gust with the same parameters as the 1 -cosine profile (i.e. $4 \mathrm{~m} / \mathrm{s}$ magnitude and $6 \mathrm{~m}$ length), was investigated 
to compare the energy extractions to that of the 1-cosine profile. Two configurations of the sinusoidal gust were investigated. The first, an upward gust followed by a downward gust, and the second a downward gust followed by an upward. The time history response for both profiles is shown in Fig. 5.9. Encountering the upward gust first produced a wing drag reduction of $6.91 \%$, while encountering the downward gust first produced a marginally reduced wing drag reduction of $6.9 \%$. The addition of a downward gust created a reduction in energy extracted when compared to the 1-cosine profile which only consists of an upward motion. This is a result of the rearward tilting of the lift vector as a result of the downward component, creating an increase in drag. This is evidenced in Fig. 5.9 when looking at both the induced drag coefficient and instantaneous drag reductions. When the gust velocity becomes negative, an increase in induced drag is noted in comparison to its steady-state value. A resulting drag increase is observed, indicated by the positive instantaneous drag reduction, which is actually a drag increase. The magnitude of the drag increase, however, is significantly less than the drag reduction, thus, still resulting in an overall drag reduction. As the negative gust velocity reaches its peak, a negative lift is produced on the wing, resulting in an additional drag reduction. Although negative gust velocities cause a rearward tilting of the lift vector at positive lift values, when lift is negative, these velocities tilt the lift vector forward, creating this drag reduction. Consequently, despite zero net air mass motion, a significant wing drag reduction is still achievable; the thrust generated from the upward gust is significant enough to outweigh the negative effects of a downward gust, resulting in an overall net thrust.

Comparing the two variations of the sinusoidal gust profiles, the downward gust followed by the upward extracted slightly less energy, as previously stated. This is due to the noticeably different elastic response, specifically the tip deflection and twist, shown in Fig. 5.9. The down-up gust saw a $15 \%$ decrease in tip deflection before returning towards its steady-state value, while the up-down gust saw a $15 \%$ increase in tip deflection from its steady-state condition. It is known from the previous study in Fig. 5.5 and from literature that increased elastic deformations enhance the energy harvesting process [7] thus, the reduction seen in the down-up gust contributes to its reduced energy extraction. The torsional response of the down-up gust, however, showed an increased pitch down response when compared to the up-down gust. The maximum pitch down response from the down-up gust was nearly 9 times its steady-state value, while the up-down gust showed a maximum twist down response of 7.5 times its steady-state value. The pitch up response of the down-up gust is also reduced in comparison to the up-down gust. The down-up gust showed an increase of only 7.46 times its steady-state value, while the up-down gust showed an increase of 9 times its steady-state condition. As previously mentioned, an increased pitch down response enables improved energy extractions. Thus, while the down-up gust suffers from reduced energy extractions as a result of reduced tip deflections, the improved pitch down 

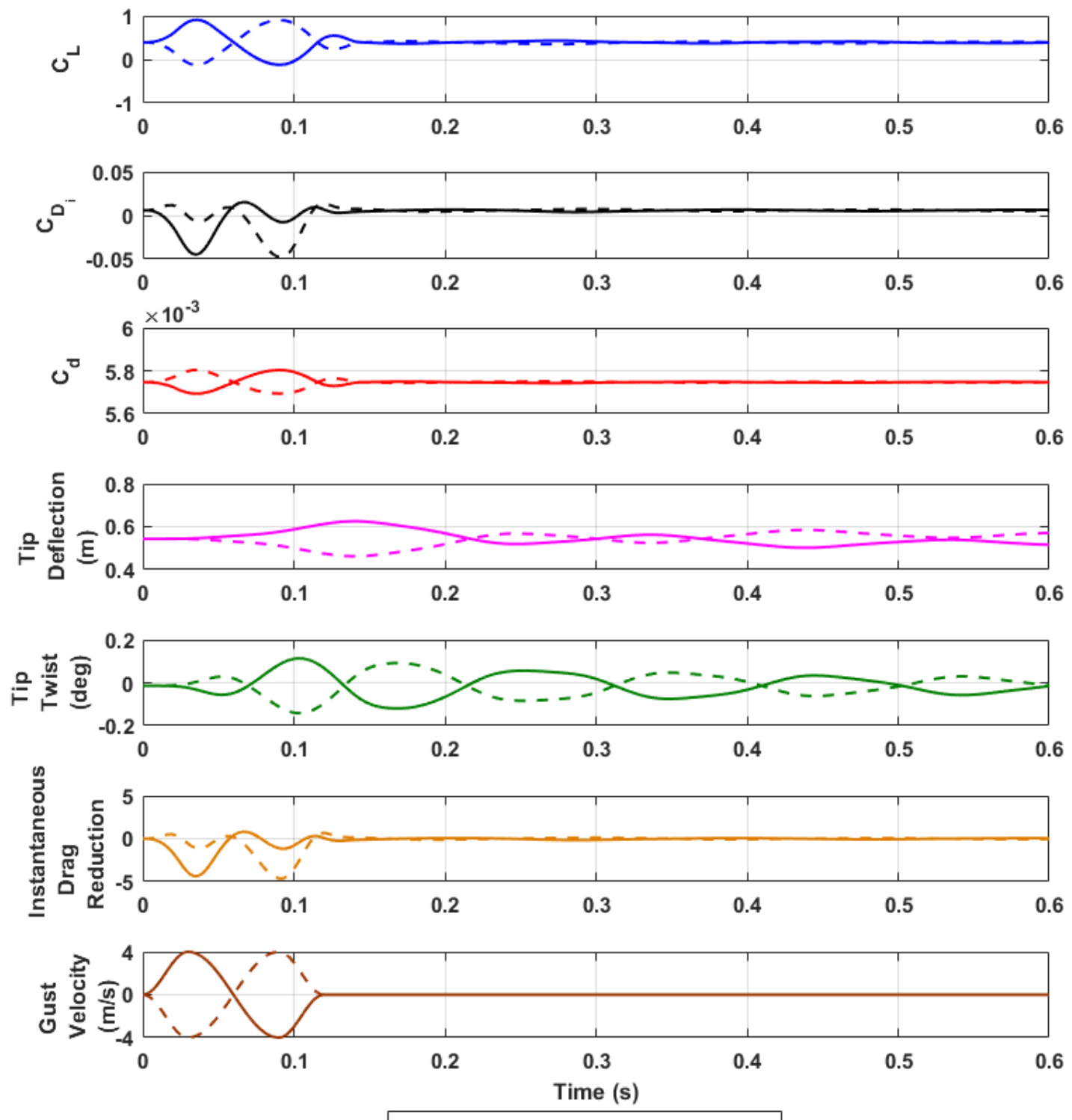

Up-Down Gust - - - Down-Up Gust

Figure 5.9: Time history of force coefficients, elastic deformations and instantaneous drag reduction for two sinusoidal gust profiles, up-down (solid) and down-up (dashed).

response in comparison to the up-down gust improves its energy extraction capabilities, thus, achieving a similar wing drag reduction. 
When performing aeroelastic tailoring studies, the effects of added stresses on the wing structure as a result of changes made are often not considered. Shifting the elastic axis towards the leading and trailing edges has a significant effect on the spanwise lift distribution, and consequently the wing root bending moment. When designing a wing structure, the wing root bending moment is a design constraint that dictates the size of structure; the structure must be able to support such moments in design and offdesign conditions. Encountering a gust provides significant changes to the wing root bending moment; these changes as a result of the gust as well as aeroelastic tailoring must be investigated. While increased energy extractions as a result of aeroelastic tailoring are possible, if these modifications result in the need to strengthen the structure in order to accommodate the increased wing root bending moments, the benefits of aeroelastic tailoring may be negated.

Figure 5.10 shows the time response of the wing root bending moment from the moment the gust is encountered. The two extreme ends of the swept elastic axis cases are plotted which showed the least and greatest energy extraction. The forward sweeping of the elastic shows an alleviating affect to the wing root bending moment, as reduced moments are seen through the duration of the gust. This is an expected result since common gust alleviation techniques involve moving the elastic axis towards the aerodynamic center. While the results of Fig. 5.10 are not entirely surprising, such an investigation should always be carried out when performing aeroelastic tailoring to determine its impact on secondary design aspects. The design of a wing structure is not a linear problem; changes that have a positive impact on one design aspect may have a significant adverse effect on a different design variable.

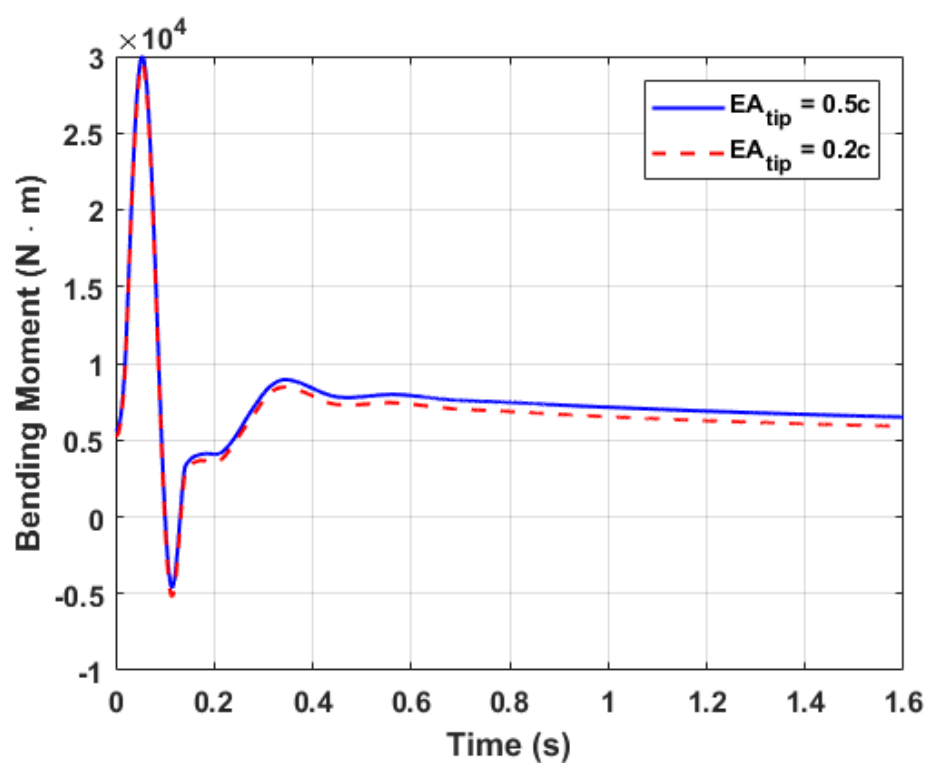

Figure 5.10: Time response of wing root bending moment for swept elastic axis. 


\subsection{Summary}

Aeroelastic tailoring was demonstrated to improve the energy extraction capabilities of an elastic wing encountering a gust in comparison to its rigid wing counterpart. These results are in agreement with those found by Mai who found that an elastic aircraft penetrating a gust has significantly more energy gains than a rigid aircraft [7]. The addition of sweep to the elastic axis provided noticeable improvements to the energy extracted when compared to an unswept elastic axis. The magnitude of the drag reductions shown here agree well with those presented by Ironside et al. [35, who, for a sinusoidal gust profile, showed nearly $10 \%$ reductions in drag. The research in Ref. 35 modelled the entire vehicle, while only the wing is modelled in this research, which could contribute to the discrepancy in results. This comparison, however, provides confidence that the presented results are within a reasonable range of what is to be expected.

The energy gains demonstrated from all cases, both straight and swept, should be considered with caution, however. While the mass axis location remained fixed in front of the elastic axis, ensuring unconditional dynamic stability, the shifting of the elastic axis relative to the aerodynamic center present concerns for static stability. Divergence is a concern with the elastic axis placed closer to the trailing edge; however, the addition of sweep towards the leading edge, in front of the aerodynamic center, eliminates the concern of divergence. Sweeping the elastic axis in front of the aerodynamic center towards the wing tip does create concerns for aileron ineffectiveness. Lift increases from deflecting the aileron can be negated by the reduction in lift from the wing twisting down; as a result, the aileron will no longer be able to provide roll control, jeopardizing the stability of the aircraft. Thus, the degree to which the elastic axis is swept must be carefully considered to avoid such instabilities.

The analysis presented also demonstrates one of the major strengths of this method, specifically in comparison to higher fidelity approaches. The ability to easily extract a force decomposition, such as a time history of profile and induced drag, to gather insight into these drag reductions is very powerful. Such a task would be very difficult to perform in a CFD-based analysis. The force decomposition allows one to determine where efforts need to be focused in order to maximize the energy extraction and allows for more insightful trends to be developed from the analysis. 


\section{Chapter 6}

\section{Conclusions}

This thesis presented an efficient tightly coupled fluid-structure interaction model for capturing the aeroelastic effects of a flexible wing encountering a gust. The aerodynamic model utilizes a higherpotential flow method that demonstrates excellent numerical robustness. An unsteady formulation of the Kutta-Joukowsky theorem was implemented to capture non-circulatory effects, improving the accuracy in capturing time dependent loads. The structural dynamics was modelled through an explicit finite difference representation of the Euler-Bernoulli dynamic beam equations. Using the coupled FSI model, the effects of aeroelastic tailoring on the gust energy extraction capabilities was investigated.

\subsection{Aeroelastic Model}

The explicit method provides an efficient means for solving the exact solution to the coupled dynamic equations of motion. Compared to other higher fidelity methods, the computational time required for a single case with the present method is significantly reduced. For a given gust case, approximately 8-9 hours of CPU time is required on a $3.5 \mathrm{GHz}$ processor; coupled CFD-CSD approaches for a similar analysis would require on the order of 36-48 hours [36. The computational time required is approximately $75 \%$ less, while the accuracy of the model is still reasonable when compared to higher fidelity approaches. This makes such an approach attractive for use in early design stages. Multiple design configurations can be investigated in the span of time it would take for one case with higher fidelity approaches. The use of numerical functions to approximate stiffness and mass distributions also allows this method to be easily used in optimization procedures. In an optimization approach the coefficients of the second order functions could be used as design variables and modified accordingly, with a specific design objective 
such as maximizing the gust efficiency.

\subsection{Implications of Aeroelastic Tailoring}

The results from the aeroelastic tailoring case studies showed that flexible configurations provide significantly increased performance gains when encountering a gust. Comparison was made between two different elastic axis orientations, straight and swept. The swept elastic axis showed improved energy extractions over the straight elastic axis cases. In both cases, shifting the elastic axis towards the aerodynamic center improved the energy extractions. Additional performance gains of approximately $0.25 \%$ were seen by sweeping the elastic axis. Multiple operating points were also investigated to determine the effect of the wing lift coefficient on the drag reduction capabilities. A maximum wing drag reduction of approximately $10.5 \%$ was seen at a lift coefficient of 0.4 ; further increases in lift coefficient showed less drag reductions over the previous cases, indicating further exploration is required to improve energy extractions at high lift coefficients. Two sinusoidal gust profiles were also investigated, showing reduced energy extractions in comparison to the 1-cosine profile. However, these results showed that despite a net zero air mass motion, a wing drag reduction of $6.9 \%$ was still possible. Less energy was shown to be extracted when encountering a down-up gust versus an up-down gust; this was due to differing elastic responses, however, the reduction in energy extracted was minimal.

While the performance gains from changing the elastic axis orientation may seem minimal, such gains can be achieved with very minimal effort. Aeroelastic tailoring only requires modification to fiber orientations when considering composite structures; a wing can be designed to support the required wing loading while also extracting energy without the need for additional hardware or control techniques. The use of aeroelastic tailoring allows for these energy gains with little to no weight penalties. When applied to long endurance aircraft such as HALE research vehicles, these energy gains can expand the range of applications of these aircraft, as well as increase the duration of missions for which they may be used. 


\section{Appendix A}

\section{Aerodynamic Module Input File}

The input file for the aerodynamics module is broken up into five different section. The first defines the analysis type. This includes specifying the wake model, steady or unsteady aerodynamics, whether a stiff or flexible wing is used and the type of gust profile. Using the stiff wing model, a regular aerodynamic analysis will be performed, while the flexible wing model will include structural dynamics. The gust mode is defined using $0,1,2$ or 3 where 0 is no gust, 1 is a sinusoidal gust, 2 is a 1-cosine gust and 3 is a sharp-edge gust. The second section defines the numerical parameters including the number of time steps, aerodynamic time step size and the convergence tolerance required for the span efficiency, if desired. This is held at zero for an aeroelastic analysis. The third section defines the freestream and flight conditions such as the angle of attack, sideslip angle, air properties and velocities. The freestream velocity as well as the both the gust strength and length are defined here; if a gust mode of 0 is selected, the inputs for gust amplitude and length are not used. The remaining two sections pertain to the lifting surface geometry. The reference area and span are used for nondimensionalizing force values into their appropriate coefficient form. Panels are used to define the wing geometry. For a simple rectangular wing, 1 panel is sufficient, however, wings with significant geometry changes are modelled with multiple panels. In defining a panel, the left and right leading edge points are defined, along with the chord and twist at their respective locations. The twist should be defined to relative to the zero-lift plane of the

given wing section. The panel definition also includes the specification of the number of spanwise and chordwise elements and the airfoil number from the data base that is to be used. 
Input file in $\mathrm{m} / \mathrm{N} / \mathrm{sec}$

Please note that the program uses equal, number and : signs as special recognizers!

Relaxed wake (yes 1, no 0):

Steady (1) or unsteady (2):

Stiff (1) or Flexible (2) Wing:

Gust Type $(0,1,2$ or 3$)$ :

Max. number of time steps:

Min. number of time steps:

Width of each time step (sec):

Convergence delta-span effic.:

timestepping)

AOA sequence:

Sideslip angle [deg]:

Density:

Kinematic viscosity:

Freestream velocity:

Gust amplitude:

Gust length:

Reference area:

Reference span:

Mean aerodynamic chord:

Aircraft weight $(\mathrm{N})$ :

Pitching Moment Coeff.:

$\begin{array}{lll}\text { flagRELAX } & = & 0 \\ \text { flagSTEADY } & = & 2 \\ \text { flagSTIFFWING } & = & 2 \\ \text { flagGUSTMODE } & = & 2\end{array}$

valMAXTIME $=1500$

valMINTIME $=0$

valDELTIME $\quad=\quad 0.0075$

valDELTAE $\quad=\quad 0.000000 \quad(0$ if only

seqALPHA $\quad=\quad 0.0$

seqBETA $\quad=\quad 0.0$

valDENSITY $=1.225$

valKINV $\quad=\quad 1.460000 \mathrm{e}-05$

valUINF $\quad=\quad 50.0$

valGUSTAMP $=4.0$

$\begin{array}{lll}\text { valGUSTL } & =6.00\end{array}$

ValAREA $\quad=\quad 32.00$

valSPAN $\quad=\quad 32.00$

valCMAC $=1$

valWEIGHT $=3434$

valCM $=-0.025$

No. of panels: valPANELS $=1$

Defines leading edge of wing, all measured in metres:

Keep vecM the same for all panels on a wing!

Panel \#:1.

Number of spanwise elements: $\quad$ vecN $\quad=10$.

Number of chordwise elements: vecM $=5$.

Airfoil number:

vecAIRFOIL $=1$

Symmetry edge $(0,1$ or 2$)$ :

$\operatorname{vecSYM}=1$;

$\begin{array}{lllll}\text { xleft } & \text { yleft } & \text { zleft } & \text { chord } & \text { epsilon } \\ 0.0000000 & 0.000000 & 0.0000000 & 1.000000 & 2.00000 \\ 0.0000000 & 16.000000 & 0.0000000 & 1.000000 & 2.00000\end{array}$




\section{Appendix B}

\section{Structural Properties Input File}

The structural input file is used to specify the second order splines which describe the stiffness and mass distributions and structural axis locations (mass and elastic) relative to the wing leading edge. For each parameter, the three coefficients of the second order function are prescribed. Numerical parameters such as the time step size and number of structural elements are also specified in this file. A flag (0 or 1) also sets whether the static aeroelastic response is determined or a full dynamic analysis is performed.

Input file $\mathrm{m} / \mathrm{N} / \mathrm{sec}$

Please note that the program uses equal, number and : signs as special recognizers!

Width of each time step (sec):

Number of spanwise structural elements:

Number of timesteps with no deflections:

Static (0) or Dynamic (1) aeroelasticity:

Stiffness Distribution $\left(\mathrm{Nm}^{\wedge} 2\right)$

Bending $\left(E^{*} \mathrm{Ix}\right)$

A Coefficient: vec EIx $=0$

B Coefficient: vec EIx $=0$

C Coefficient: vec_EIx $=750000$

Torsion $\left(\mathrm{G}^{*} \mathrm{Jt}\right)$ :

A Coefficient: vec_GJt $=0$

B Coefficient: vec_GJt $=0$

C Coefficient: vec_GJt $=750000$

$\begin{array}{lll}\text { valSDELTIME } & = & 0.00075 \\ \text { valNSELE } & = & 20 \\ \text { valSTIFFSTEPS } & = & 20 \\ \text { flagSTATIC } & = & 1\end{array}$


Geometry

Elastic Axis (m):

A Coefficient: vec_EA $=0$

B Coefficient: vec_EA $=0$

C Coefficient: vec_EA $=0.25$

Center of Gravity (m):

A Coefficient: vec_CG $=0$

B Coefficient: vec_CG $=0$

C Coefficient: vec_CG $=0.20$

Mass Distribution

Mass Moment of Inertia (kgm):

A Coefficient: vec_Jt $=0$

B Coefficient: vec_Jt $=0$

C Coefficient: vec_Jt $=2.0$

Linear Mass $(\mathrm{kg} / \mathrm{m})$ :

A Coefficient: vec_LM $=0$

B Coefficient: vec_LM $=0$

C Coefficient: vec_LM $=5.0$ 


\section{Bibliography}

[1] Arioli, G. and Gazzola, F., "Torsional instability in suspension bridges: The Tacoma Narrows Bridge case," Communications in Nonlinear Science and Numerical Simulation, Vol. 42, 2017, pp. 342-357.

[2] Noll, T. E., Brown, J. M., Perez-davis, M. E., Ishmael, S. D., Tiffany, G. C., and Gaier, M., "Investigation of the Helios Prototype Aircraft Mishap," Tech. rep., 2004.

[3] Tanaka, M. and Bercin, A. N., "Free vibration solution for uniform beams of nonsymmetrical cross section using Mathematica," Computers and Structures, Vol. 71, No. 1, 1999.

[4] Melville, M., Bramesfeld, G., Alighanbari, H., and Kolaei, A., "The Effects of Elastic Deformation on HALE Aircraft Flight Performance," 63rd CASI Aeronautics Conference, Toronto, 2017.

[5] Katzmayr, R., "Effect of periodic changes of angle of attack on behavior of airfoils," 1922, pp. NACA-TM-147.

[6] Jones, K. D., Dohring, C. M., and Platzer, M. F., "Experimental and Computational Investigation of the Knoller-Betz Effect,” AIAA Journal, Vol. 36, No. 7, 1998, pp. 1240-1246.

[7] Mai, H. U., "The Effect of Aeroelasticity Upon Energy Retrieval Of A Sailplane Penetrating A Gust.pdf," XIXth Congreess of OSTIV, 1985.

[8] Phillips, W. H., "Propulsive effects due to flight through turbulence," Journal of Aircraft, Vol. 12, No. 7,1975 , pp. $624-626$.

[9] Bruni, C., Gibert, J., Frulla, G., Cestino, E., and Marzocca, P., "Energy harvesting from aeroelastic vibrations induced by discrete gust loads," Journal of Intelligent Material Systems and Structures, Vol. 28, No. 1, 2017, pp. 47-62.

[10] Langelaan, J. W., "Gust Energy Extraction for Mini and Micro Uninhabited Aerial Vehicles," Journal of Guidance, Control, and Dynamics, Vol. 32, No. 2, 2009, pp. 464-473. 
[11] Shirk, M. H., Hertz, T. J., and Weisshaar, T. A., "Aeroelastic tailoring - Theory, practice, and promise," Journal of Aircraft, Vol. 23, No. 1, 1986, pp. 6-18.

[12] Weisshaar, T., Nam, C., and Batista-Rodriguez, A., "Aeroelastic tailoring for improved UAV performance," 39th AIAA/ASME/ASCE/AHS/ASC Structures, Structural Dynamics, and Materials Conference and Exhibit, 1998.

[13] Lupp, C. A. and Cesnik, C. E., "Aeroelastic Tailoring for Maximizing Sailplane Average CrossCountry Speed," AIAA Atmospheric Flight Mechanics Conference, 2015.

[14] Francois, G., Cooper, J. E., and Weaver, P. M., "Aeroelastic Tailoring using Rib / Spar Orientations : Experimental Investigation," 56th AIAA/ASMe/ASCE/AHS/SC Structures, Structural Dynamics, and Material Conference, Kissimmee, Florida, USA, 2015.

[15] Smith, M., Patil, M., and Hodges, D., "CFD-based analysis of nonlinear aeroelastic behavior of high-aspect ratio wings," 19th AIAA Applied Aerodynamics Conference, 2001.

[16] Raveh, D. E., "Gust-Response Analysis of Free Elastic Aircraft in the Transonic Flight Regime," Journal of Aircraft, Vol. 48, No. 4, 2011, pp. 1204-1211.

[17] Reimer, L., Ritter, M., Heinrich, R., and Kr, W., "CFD-based Gust Load Analysis for a Free-flying Flexible Passenger Aircraft in Comparison to a DLM-based Approach," AIAA Aviation 2015, 2015, pp. 1-17.

[18] Donaldson, B. K., Introduction to Structural Dynamics, Cambridge University Press, 2006.

[19] Ironside, D., Bramesfeld, G., and Schwochow, J., "Modeling of Wing Drag Reductions Due to Structural Dynamics in Atmospheric Gusts," 28th AIAA Applied Aerodynamics Conference, 2010.

[20] Subbaraj, K. and Dokainish, M. A., "A Survey of Direct Time-Integration Methods in Computational Structural DynamicsII. Implicit methods," Vol. 32, No. 6, 1989, pp. 1387-1401.

[21] Newmark, N., "A Method of Computation for Structural Dynamics," 1959.

[22] Wilson, E. L., Farhoomand, I., and Bathe, K. J., "Nonlinear Dynamic Analysis of Complex Structures," Earthquake Engineering and Structural Dynamics, Vol. 1, No. 3, 1972, pp. 241-252.

[23] Houbolt, J. C., "A Recurrence Matrix Solution for the Dynamic Response of Aircraft in Gusts," Report 1010, 1951.

[24] Bramesfeld, G., A Higher Order Vortex-Lattice Method with a Force-Free Wake, Ph.D. thesis, 2006. 
[25] Wang, Z., Chen, P. C., Liu, D., and Mook, D., "Nonlinear Aeroelastic Analysis for A HALE Wing Including Effects of Gust and Flow Separation," 48th AIAA/ASME/ASCE/AHS/ASC Structures, Structural Dynamics, and Materials Conference, 2007.

[26] Schirra, J. C., Watmuff, J. H., and Bauschat, J. M., "Highly non-planar Lifting Systems: A relative assessment of existing Potential-Methodologies to accurately estimate the Induced Drag," 32nd AIAA Applied Aerodynamics Conference, No. 3095-3109.

[27] Melville, M., "Design of Wings with Improved Gust Response," Tech. rep., Ryerson University, 2016.

[28] Cole, J. A., A Higher-Order Free-Wake Method for Propeller-Wing Systems, Ph.D. thesis, Pennsylvania State University, 2016.

[29] Hallissy, B. P. and Cesnik, C. E. S., "High-fidelity Aeroelastic Analysis of Very Flexible Aircraft," 52nd AIAA/ASME/ASCE/AHS/ASC Structures, Structural Dynamics and Materials Conference, 2011.

[30] Thomas, F., Fundamentals of Sailplane Design, College Park Press, 1999.

[31] Banerjee, J., "Explicit Frequency Equation and Mode Shapes of a Cantilever Beam Coupled in Bending and Torsion," Journal of Sound and Vibration, Vol. 224, No. 2, 1999, pp. 267-281.

[32] Yeung, A., Wind Gust Measuring at Low Altitude Using an Unmanned Aerial System, Master's thesis, Ryerson University, 2017.

[33] Wang, Z. c., Chen, P. c., Liu, D. c., Mook, D. d., and Patil, M. e., "Time domain nonlinear aeroelastic analysis for HALE wings," Collection of Technical Papers - AIAA/ASME/ASCE/AHS/ASC Structures, Structural Dynamics and Materials Conference, Vol. 1, 2006, pp. 488-506.

[34] Melville, M., Kolaei, A., Bramesfeld, G., and Alighanbari, H., "An Efficient Model for Aeroelastic Tailoring of Aircraft Wings Under Gust Loads," 2018 Aerospace Sciences Meeting, 2018.

[35] Bramesfeld, G., Ironside, D. J., and Schwochow, J., "Simplified modeling of wing-drag reduction due to structural dynamics and atmospheric gusts," Collection of Technical Papers - AIAA Applied Aerodynamics Conference, 2008.

[36] Combes, T. P., Malik, A. S., Bramesfeld, G., and McQuilling, M. W., "Efficient Fluid-Structure Interaction Method for Conceptual Design of Flexible, Fixed-Wing Micro-Air-Vehicle Wings," AIAA Journal, Vol. 53, No. 6, 2015, pp. 1442-1454. 
